\title{
LOGARITHMIC DEFORMATIONS OF NORMAL CROSSING ENRIQUES SURFACES IN CHARACTERISTIC TWO
}

\author{
STEFAN SCHRÖER
}

25 March 2001

\begin{abstract}
Working in characteristic two, I classify nonsmooth Enriques surfaces with normal crossing singularities. Using Kato's theory of logarithmic structures, I show that such surfaces are smoothable and lift to characteristic zero, provided they are d-semistable.
\end{abstract}

\section{INTRODUCTION}

Smooth Enriques surfaces occupy a special position in the classification of surfaces: They are similar to rational surfaces but have Kodaira dimension zero. Here we shall study Enriques surfaces that are normal crossing rather than smooth: What they are; how they look like; their fibrations or embeddings; and whether they deform to smooth surfaces or lift to characteristic zero. Since $p=2$ is the most exciting prime with respect to Enriques surfaces and to surface normal crossings as well, I shall restrict my attention to characteristic two.

Little seems to be known on the relation of Enriques surfaces in characteristic two and characteristic zero. However, Ekedahl and Shepherd-Barron recently announced that smooth Enriques surface in characteristic two lift to characteristic zero. Our main results is the following Theorem:

Theorem. Nonsmooth d-semistable Enriques surfaces with normal crossings are smoothable and lift to characteristic zero.

Note that this does not require projectivity. The result might be useful for the construction of complete moduli spaces over $\operatorname{Spec}(\mathbb{Z})$ for Enriques surfaces.

Working over the complex numbers, Kulikov [18] started the study of simple normal crossing K3 and Enriques surfaces. Using Hodge theory, he obtained a classification of such surfaces. Taking the peculiarities of characteristic $p=2$ into account, I shall give a similar classification. Criteria for classification are: Structure of Picard scheme (classical, ordinary, or supersingular); multiplicity of singularities (type II or type III); and nature of irreducible components (simple or nonsimple).

Friedman's paper [9] is fundamental for the theory of smoothings. Working in the complex analytic category, he showed that precisely the d-semistable K3 surfaces from Kulikov's list deform to smooth K3 surfaces. Using logarithmic structures, Kawamata and Namikawa [17] simplified Friedman's proof. Little attention, however, was paid to positive characteristics.

1991 Mathematics Subject Classification. 14D15, 14J28, 14L20. 
STEFAN SCHROEER

The theory of logarithmic structures goes back to Fontaine and Illusie and was developed by K. Kato [16]. I shall apply the work of F. Kato [15 on log deformations to d-semistable Enriques surfaces. We shall see that the log deformation functor is formally smooth for classical and ordinary Enriques surfaces. In contrast, supersingular surfaces are obstructed.

In the category of schemes the existence of formal smoothings does not imply the existence of algebraic smoothings. In light of Grothendieck's Existence Theorem, algebraization hinges on the presence of an formal ample sheaf. There are no obstructions for for classical Enriques surfaces. For type III Enriques surfaces, however, we first have to pass to minus-one-form and check that a sufficiently large part of the versal deformation is algebraizable.

Acknowledgement. I wish to thank Bernd Siebert and Hubert Flenner for stimulating discussions.

\section{EnRIQUes SURFACES WITH NORMAL CROSSINGS}

Fix a ground field $k$, for the moment of arbitrary characteristic $p \geq 0$. To study degenerations of smooth Enriques surfaces we have to decide on a suitable notion of singular Enriques surfaces.

Definition 1.1. A proper $k$-surface $X$ with $k=\Gamma\left(X, \mathcal{O}_{X}\right)$ is called a normal crossing Enriques surface if the singularities of $X$ are normal crossings, and the canonical class $K_{X}$ is numerically trivial, and $\chi\left(\mathcal{O}_{X}\right)=1$ holds.

For smooth Enriques surfaces, this coincides with the usual definition $K_{X} \equiv 0$ and $b_{2}(X)=10$ (compare $\left.\mid 6\right]$, pp. 72-74). There are interesting degenerations of Enriques surfaces with $K_{X} \not \equiv 0$ (so-called flower pot degenerations). For this paper, however, I am content with the case $K_{X}$ numerically trivial.

Note that normal crossing is a local condition, such that the irreducible components of $X$ might be nonsmooth. Call $X$ simple if all its irreducible components are smooth, and nonsimple otherwise.

By definition, the singularities are normal crossings if for each point $x \in X$ there is an isomorphism $\mathcal{O}_{X, x}^{\wedge} \simeq \kappa(x)\left[\left[T_{1}, T_{2}, T_{3}\right]\right] /\left(T_{1} \ldots T_{l}\right)$. The integer $l \in\{1,2,3\}$ is called the multiplicity of $x \in X$. A point $x \in X$ with multiplicity $l=1$ is smooth. Points with multiplicity $l=2$ or $l=3$ are called double points or triple points, respectively. We shall say that $X$ is an Enriques surface of type $I I$ if it is a normal crossing Enriques surface with double points but without triple points; and of type $I I I$ if it contains triple points.

Another way to distinguish normal crossing Enriques surfaces involves the Picard scheme. Let $\operatorname{Pic}^{\tau}(X)$ be the group of numerically trivial line bundles and $\operatorname{Pic}_{X}^{\tau}$ be the corresponding group scheme. These objects behave like in the smooth case:

Proposition 1.2. Suppose $X$ is a normal crossing Enriques surface. Then $\operatorname{Pic}_{X}^{\tau}$ is an affine group scheme of length 2. Furthermore, $\operatorname{Pic}^{\tau}(X)$ is generated by $K_{X}$.

Proof. Note that a numerically trivial invertible $\mathcal{O}_{X}$-module is trivial if and only if it has a nonzero section, because $X$ is connected and reduced. Suppose $\mathcal{L} \neq \mathcal{O}_{X}$ is such a line bundle. By Riemann-Roch, $\chi(\mathcal{L})=\chi\left(\mathcal{O}_{X}\right)=1$. On the other hand,

$$
\chi(\mathcal{L}) \leq h^{0}(\mathcal{L})+h^{0}\left(\mathcal{L}^{\vee} \otimes \omega_{X}\right)=h^{0}\left(\mathcal{L}^{\vee} \otimes \omega_{X}\right),
$$

consequently $\mathcal{L} \simeq \omega_{X}$. Hence, if $\operatorname{Pic}^{\tau}(X)$ is nontrivial, it is generated by $K_{X}$ and has order two. 
In light of this, the connected component $\operatorname{Pic}_{X}^{0} \subset \operatorname{Pic}_{X}^{\tau}$ is discrete. Its Lie algebra is $H^{1}\left(X, \mathcal{O}_{X}\right)$. There are two possibilities: If $K_{X} \neq 0$, then $h^{1}\left(\mathcal{O}_{X}\right)=0$, so $\mathrm{Pic}_{X}^{0}=0$ and $\mathrm{Pic}_{X}^{\tau}=\mathbb{Z} / 2 \mathbb{Z}$. If $K_{X}=0$, then $\operatorname{Pic}_{X}^{0}=\operatorname{Pic}_{X}^{\tau}$. Moreover, $h^{1}\left(\mathcal{O}_{X}\right)=1$, so $\operatorname{Pic}_{X}^{0}$ has length two. In both cases we see that $\operatorname{Pic}_{X}^{\tau}$ is an affine group scheme of length two.

Besides the étale group scheme $\mathbb{Z} / 2 \mathbb{Z}=\operatorname{Spec}\left[T \mid T^{2}=T\right]$, there are two radical (that is, purely inseparable) group schemes of length two in characteristic $p=2$ :

$$
\mu_{2}=\operatorname{Spec} k\left[T \mid T^{2}=1\right] \quad \text { and } \quad \alpha_{2}=\operatorname{Spec} k\left[T \mid T^{2}=0\right] .
$$

Note that $\alpha_{2}$ and $\mathbb{Z} / 2 \mathbb{Z}$ are unipotent group schemes, whereas $\mu_{2}$ is a multiplicative group scheme. We shall say that $X$ is a classical Enriques surface if $X$ is a normal crossing Enriques surface with $\operatorname{Pic}_{X}^{\tau}=\mathbb{Z} / 2 \mathbb{Z}$; ordinary if $\operatorname{Pic}_{X}^{\tau}=\mu_{2}$; and supersingular if $\operatorname{Pic}_{X}^{\tau}=\alpha_{2}$.

\section{How do NORMal CROSSING ENRIQUeS SURFACES LOOK LIKE?}

Fix an algebraically closed ground field $k$ of characteristic $p=2$, and let $X$ be an Enriques surface of type II or III. The task now is to determine the structure of such surfaces. The idea is to reconstruct $X$ from its normalization via gluing.

Let $\nu: S \rightarrow X$ be the normalization, $C \subset S$ the reduced ramification locus of the normalization map, and $D \subset X$ the reduced singular locus. Then $S$ is a smooth surface, $C \subset S$ is a normal crossing divisor, and $D$ is a seminormal curve. The commutative diagram

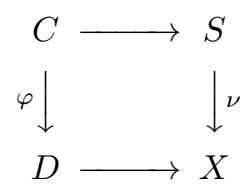

is cartesian and cocartesian. Hence we can recover $X$ from the smooth surface $S$ and the gluing map $\varphi: C \rightarrow D$. The diagram yields a short exact sequence

$$
1 \longrightarrow \mathcal{O}_{X}^{\times} \longrightarrow \mathcal{O}_{S}^{\times} \oplus \mathcal{O}_{D}^{\times} \longrightarrow \mathcal{O}_{C}^{\times} \longrightarrow 1
$$

which in turn gives a long exact sequence of abelian sheaves

$$
H^{0}\left(\mathcal{O}_{C}^{\times}\right) \longrightarrow \operatorname{Pic}(X) \longrightarrow \operatorname{Pic}(S) \oplus \operatorname{Pic}(D) \longrightarrow \operatorname{Pic}(C) \longrightarrow H^{2}\left(\mathcal{O}_{X}^{\times}\right)
$$

The relative dualizing sheaf $\omega_{S / X}$ coincides with the conductor ideal of the inclusion $\mathcal{O}_{X} \subset \nu_{*}\left(\mathcal{O}_{S}\right)$, so we have

$$
K_{S}=K_{S / X}+\nu^{*}\left(K_{X}\right) \equiv-C \quad \text { and } \quad 2 K_{S}=-2 C .
$$

Decompose $S=S_{1} \cup \ldots \cup S_{n}$ into connected components, and let $X=X_{1} \cup \ldots \cup$ $X_{n}$ be the corresponding irreducible components. Then $2 K_{S_{i}}=-2 C_{S_{i}}$. By the classification of surfaces [20], each component $S_{i}$ is ruled.

The ramification locus $C \subset S$ is a divisor with normal crossings. Its global structure is easy: The adjunction formula gives $2 K_{C}=0$. It follows $K_{C}=0$, so $C$ is a disjoint union of elliptic curves and cycles of rational curves. Here a cycle of rational curves means a seminormal curve isomorphic to $\mathbb{P}^{1} \times \mathbb{Z} / m \mathbb{Z}$ (with $m \geq 1$ ) modulo the relation $(\infty, i) \sim(0, i+1)$ for $i \in \mathbb{Z} / m \mathbb{Z}$.

Proposition 2.1. Suppose the component $S_{i} \subset S$ is a rational surface. Then the ramification locus $S_{i} \cap C$ is either an elliptic curve or a cycle of rational curves. 
STEFAN SCHROEER

Proof. Set $C_{i}=S_{i} \cap C$. Then $K_{S_{i}}=-C_{i}$ because $\operatorname{Pic}\left(S_{i}\right)$ is torsion free. The exact sequence

$$
0 \longrightarrow H^{0}\left(S_{i}, \mathcal{O}_{S_{i}}\right) \longrightarrow H^{0}\left(C_{i}, \mathcal{O}_{C_{i}}\right) \longrightarrow H^{1}\left(S_{i}, \omega_{S_{i}}\right)
$$

and $H^{1}\left(S_{i}, \mathcal{O}_{S_{i}}\right)=0$ implies that $C_{i}$ is connected, so $C_{i}$ is as desired.

To proceed, we need a fact on elliptic curves in characteristic $p=2$.

Lemma 2.2. For an elliptic curve $E$ over $k$, the following are equivalent.

(i) The Frobenius map $\mathrm{Fr}^{*}: H^{1}\left(E, \mathcal{O}_{E}\right) \rightarrow H^{1}\left(E, \mathcal{O}_{E}\right)$ is zero.

(ii) There is an inclusion of group schemes $\alpha_{2} \subset E$.

(iii) $E$ has a Weierstrass equation of the form $y^{2}+y=x^{3}$.

(iv) The $j$-invariant is $j(E)=0$.

(v) The group $E(k)$ has no 2-torsion.

(vi) $\operatorname{Aut}(E)$ is a group of order 24.

For a proof, see Silverman [24], Appendix A. Note that each elliptic curve over $k$ has a Weierstrass equation $y^{2}+\alpha x y+y=x^{3}$ with $\alpha^{3} \neq 1$, which is called Deuring normal form. The corresponding $j$-invariant is $j=\alpha^{12} /\left(\alpha^{3}-1\right)$. Condition (i) means that $E$ has Hasse invariant zero. Such elliptic curves are called supersingular. We shall see that supersingular elliptic curves are closely related to supersingular Enriques surfaces.

Proposition 2.3. Suppose the component $S_{i} \subset S$ is a nonrational surface. Then there is a unique ruling $f_{i}: S_{i} \rightarrow B_{i}$ over an elliptic curve $B_{i}$. The curve $C \cap S_{i}$ is either the union of two disjoint sections, or an elliptic curve double-covering $B_{i}$. Moreover, $\left.\nu^{*}\left(K_{X}\right)\right|_{S_{i}} \neq 0$ if and only if $C \cap S_{i} \rightarrow B_{i}$ is radical and $j\left(B_{i}\right) \neq 0$.

Proof. Set $C_{i}=C \cap S_{i}$. The Albanese morphism yields a unique ruling $f_{i}: S_{i} \rightarrow B_{i}$ over a smooth nonrational curve $B_{i}$. The Hurwitz Formula applied to $C_{i} \rightarrow B_{i}$ tells us that $B_{i}$ is elliptic. By Lüroth's Theorem, $C_{i}$ must be a disjoint union of elliptic curves. By the adjunction formula, the projection $C_{i} \rightarrow B_{i}$ has degree 2 , so $C_{i}$ is either the union of two disjoint sections or an elliptic curve double-covering $B_{i}$.

It remains to verify the assertion concerning the numerically trivial invertible $\mathcal{O}_{S_{i}}$-module $\mathcal{L}_{i}=\left.\nu^{*}\left(\omega_{X}\right)\right|_{S_{i}}$. The exact sequence

$$
0 \longrightarrow \omega_{S_{i}} \otimes \mathcal{L}_{i}^{\vee} \longrightarrow \mathcal{O}_{S_{i}} \longrightarrow \mathcal{O}_{C_{i}} \longrightarrow 0
$$

gives an exact sequence

$$
H^{1}\left(S_{i}, \mathcal{O}_{S_{i}}\right) \longrightarrow H^{1}\left(C_{i}, \mathcal{O}_{C_{i}}\right) \longrightarrow H^{2}\left(S_{i}, \omega_{S_{i}} \otimes \mathcal{L}_{i}^{\vee}\right) \longrightarrow 0 .
$$

Note that $H^{1}\left(\mathcal{O}_{B_{i}}\right) \rightarrow H^{1}\left(\mathcal{O}_{S_{i}}\right)$ is bijective, and $H^{2}\left(\omega_{S_{i}} \otimes \mathcal{L}_{i}^{\vee}\right) \simeq H^{0}\left(\mathcal{L}_{i}\right)$. If $C_{i}$ is the union of two disjoint section, then $h^{1}\left(\mathcal{O}_{C_{i}}\right)=2$, hence $\mathcal{L}_{i}$ has a nontrivial section, so $\left.\nu^{*}\left(K_{X}\right)\right|_{S_{i}}=0$.

Now suppose that $C_{i}$ is connected. Choosing suitable group structures, we may assume that $C_{i} \rightarrow B_{i}$ is a homomorphism of group schemes. We obtain an exact sequence

$$
0 \longrightarrow G \longrightarrow C_{i} \longrightarrow B_{i} \longrightarrow 0,
$$

where the kernel $G$ is one of $\mu_{2}, \alpha_{2}$ or $\mathbb{Z} / 2 \mathbb{Z}$. Applying the functor $\operatorname{Ext}^{*}\left(\cdot, \mathbb{G}_{m}\right)$ to the preceding exact sequence, we obtain another exact sequence

$$
0 \longrightarrow D(G) \longrightarrow \operatorname{Pic}_{B_{i}}^{0} \longrightarrow \operatorname{Pic}_{C_{i}}^{0} \longrightarrow 0
$$


Here $D(G)=\operatorname{Hom}\left(G, \mathbb{G}_{m}\right)$ is the Cartier dual of $G$. We shall distinguish two cases: First, suppose $G$ is either $\mathbb{Z} / 2 \mathbb{Z}$ or $\alpha_{2}$. Then the Cartier dual $D(G)$ is infinitesimal, so the map $H^{1}\left(\mathcal{O}_{B_{i}}\right) \rightarrow H^{1}\left(\mathcal{O}_{C_{i}}\right)$ is zero, hence $\mathcal{L}_{i}^{\vee}$ has a nontrivial section. Using the exact sequence (2) we conclude $\left.\nu^{*}\left(K_{X}\right)\right|_{S_{i}}=0$. Second, suppose $G=\mu_{2}$ is multiplicative. Then $D(G)=\mathbb{Z} / 2 \mathbb{Z}$ is étale, so the map $H^{1}\left(\mathcal{O}_{B_{i}}\right) \rightarrow H^{1}\left(\mathcal{O}_{C_{i}}\right)$ is bijective. We deduce $\left.\nu^{*}\left(K_{X}\right)\right|_{S_{i}} \neq 0$.

Finally, note that, if $C_{i} \rightarrow B_{i}$ is radical (that is, purely inseparable), the curves $C_{i}$ and $B_{i}$ are isomorphic as $\mathbb{Z}$-schemes without $k$-structures, and the projection $C_{i} \rightarrow B_{i}$ is nothing but the Frobenius morphism Fr: $B_{i} \rightarrow B_{i}$. Using Lemma 2.2, we see that $j\left(B_{i}\right) \neq 0$ holds if and only if $G=\mu_{2}$.

We shall use a bicoloured graph to describe the combinatorics of type II surfaces. The vertices and edges of this graph are as follows:

- White vertices: $\pi_{0}(S)$.

- Black vertices: $\pi_{0}(D)$.

- Edges: $\pi_{0}(C)$.

Here $\pi_{0}($.$) denotes the set of connected components. An edge C^{\prime} \subset C$ connects a white vertex $S^{\prime} \subset S$ with a black vertex $D^{\prime} \subset D$ if and only if $C^{\prime} \subset S^{\prime}$ and $\varphi\left(C^{\prime}\right) \subset D^{\prime}$.

Remark 2.4. The preceding definition makes sense for any seminormal scheme $X$, with $C \subset S$ the ramification locus of the normalization $\nu: S \rightarrow X$, and $D \subset X$ support of $\nu_{*}\left(\mathcal{O}_{S}\right) / \mathcal{O}_{X}$. For seminormal curves, the construction is due to Deligne and Rapoport ([7], section 3.5).

Theorem 2.5. Suppose $X$ is a nonsimple Enriques surface of type II. Then the bicoloured graph $\Gamma(X)$ has the form:

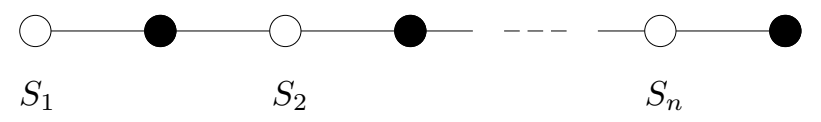

The first component $S_{1}$ is a rational surface, whereas the subsequent components $S_{2}, \ldots, S_{n}$ are elliptic ruled. Furthermore, $X$ is ordinary.

Proof. The number of rational components $S_{i} \subset S$ equals $\chi\left(\mathcal{O}_{S}\right)$. Since $X$ is triplepoint-free, both the ramification locus $C$ and the singular locus $D$ are disjoint unions of elliptic curves. Hence we have

$$
1=\chi\left(\mathcal{O}_{X}\right)=\chi\left(\mathcal{O}_{S}\right)+\chi\left(\mathcal{O}_{D}\right)-\chi\left(\mathcal{O}_{C}\right)=\chi\left(\mathcal{O}_{S}\right) .
$$

Consequently, there is precisely one rational component, say $S_{1} \subset S$. It has at most one neighbor, because $C_{1}$ is connected by Proposition 2.1. For $2 \leq i \leq n$, the curve $C_{i}$ has at most two connected components, so the graph $\Gamma(X)$ is a chain. By assumption, $X$ has a nonsmooth component, hence the last vertex is black.

It remains to check that $X$ is ordinary. Let $D^{\prime} \subset D$ be the connected component corresponding to the rightmost black vertex, and let $C^{\prime} \subset C$ be its preimage. Choosing suitable base points, we may assume that the gluing is given by an exact sequence

$$
0 \longrightarrow \mathbb{Z} / 2 \mathbb{Z} \longrightarrow C^{\prime} \longrightarrow D^{\prime} \longrightarrow 0
$$

of elliptic curves with group structure. The functor $\operatorname{Ext}^{*}\left(\cdot, \mathbb{G}_{m}\right)$ gives a dual exact sequence

$$
0 \longrightarrow D(\mathbb{Z} / 2 \mathbb{Z}) \longrightarrow \mathrm{Pic}_{D^{\prime}}^{0} \longrightarrow \mathrm{Pic}_{C^{\prime}}^{0} \longrightarrow 0 \text {. }
$$


Using the long exact sequence in (11), you easily infer $\mu_{2} \subset \operatorname{Pic}_{X}^{\tau}$. Consequently $X$ is ordinary.

Example 2.6. The case $n=1$ is allowed: Take $S=\mathbb{P}^{2}$, and let $C \subset S$ be a smooth cubic. The Jacobian $\mathrm{Pic}_{C}^{0}$ acts freely on $C$. Choose an invertible $\mathcal{O}_{C}$-module of order two, and let $\iota: C \rightarrow C$ be the corresponding free involution, say with quotient $D=C / \iota$. Now $X=S \coprod_{C} D$ is an irreducible Enriques surface of type II.

Theorem 2.7. Suppose $X$ is a simple Enriques surface of type II. Then the bicoloured graph $\Gamma(X)$ has the form:

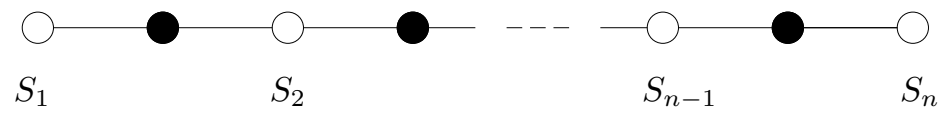

The first component $S_{1}$ is rational, whereas $S_{2}, \ldots, S_{n}$ are elliptic ruled. Moreover, if $f_{n}: S_{n} \rightarrow B_{n}$ is the ruling on the last component and $C_{n} \subset C$ is the component corresponding to the last edge, we have the following:

(i) The surface $X$ is ordinary if and only if $f_{n}: C_{n} \rightarrow B_{n}$ is étale.

(ii) $X$ is classical if and only if the projection $C_{n} \rightarrow B_{n}$ is radical and $j\left(B_{n}\right) \neq 0$.

(iii) $X$ is supersingular if and only if $C_{n} \rightarrow B_{n}$ is radical and $j\left(B_{n}\right)=0$.

Proof. You verify the first two assertions as in the preceding proof. For the last statement, consider for example the case that $C_{n} \rightarrow B_{n}$ is radical with $j\left(B_{n}\right)=0$. Then $C_{n}$ and $B_{n}$ are isomorphic as $\mathbb{Z}$-schemes without $k$-structures, so we easily obtain an exact sequence

$$
0 \longrightarrow \alpha_{2} \longrightarrow \operatorname{Pic}_{S_{n}}^{0} \longrightarrow \operatorname{Pic}_{C_{n}}^{0} \longrightarrow 0
$$

Using the exact sequence in (1), we infer $\alpha_{2} \subset \operatorname{Pic}_{X}^{\tau}$, so $X$ is supersingular. You handle the remaining cases in a similar way.

The next task is to treat type III surfaces. We shall describe their combinatorics in terms of cell decompositions $\Pi(X)$ of compact real 2-manifolds as follows: The 1-skeleton of the cell decomposition is the bicoloured graph $\Gamma(D)$ attached to the seminormal curve $D$ as in Remark 2.4. The 2-cells correspond to $\pi_{0}(S)$. It remains to specify attaching maps. We shall see that each $C_{i}=S_{i} \cap C$ is a cycle of rational curves, so $\Gamma\left(C_{i}\right)$ triangulates the real circle $\mathbb{R} / \mathbb{Z}$. For each 2 -cell corresponding to a connected component $S_{i} \subset S$, identify its boundary circle with $\Gamma\left(C_{i}\right)$ and use the canonical map $\Gamma\left(C_{i}\right) \rightarrow \Gamma(D)$ for attaching the 2-cell to the 1-skeleton $\Gamma(D)$. You directly check that this yields a cell decomposition $\Pi(X)$ of a compact real 2-manifold.

Remarks 2.8. (i) The cell decomposition $\Pi(X)$ is nothing but a dessin d'enfant, except that we do not require orientability. Such objects were introduced by Grothendieck [13] in his anabelian study of the absolute Galois group $\operatorname{Aut}(\overline{\mathbb{Q}})$.

(ii) The cell decomposition is uniquely determined by a regular neighborhood of the 1-skeleton $\Gamma(D) \subset \Pi(X)$, which is a ribbon graph. You can specify ribbon graphs in the following way: Choose an immersion of $\Gamma(D)$ into the real plane and mark those edges whose twisted ribbons shall have a single twist. For an illustration, see Example 2.10.

Now we are ready to describe surfaces with triple points. The following result was obtained by Kulikov [18] for simple normal crossings in the complex analytic case via Hodge theoretic arguments. 
ENRIQUES SURFACES IN CHARACTERISTIC TWO

Theorem 2.9. Suppose $X$ is a type III Enriques surface. Then the real 2-manifold underlying $\Pi(X)$ is the Klein bottle $\mathbb{R P}^{2}$. Each component $S_{i} \subset S$ is rational, and each ramification curve $C \cap S_{i}$ is a cycle of rational curves. Furthermore, $X$ is ordinary.

Proof. Since $X$ contains a triple point, there is at least on cycle of rational curves inside $C$. The component $S_{j}$ containing it is rational, and $C \cap S_{j}$ is a cycle of rational curves. Consequently, each component $S_{i}$ intersecting $S_{j}$ is also rational and $C_{i}$ is a cycle of rational curves. Since $X$ is connected, this holds for all components $S_{i}$. The exact sequence in (11) yields an inclusion $\operatorname{Pic}_{X}^{\tau} \subset \operatorname{Pic}_{D}^{0}$. Since $D$ is seminormal, $\mathrm{Pic}_{D}^{0}$ is a torus, so the subgroup $\operatorname{Pic}_{X}^{\tau}$ is multiplicative. Since $\mathbb{Z} / 2 \mathbb{Z}$ and $\alpha_{2}$ are not multiplicative in characteristic two, we infer that $\operatorname{Pic}_{X}^{\tau}=\mu_{2}$, so $X$ is ordinary.

It remains to determine the real 2-manifold underlying $\Pi(X)$. We do this by calculating the Euler characteristic $\chi=v-e+f$. The number of faces $f$ equals the number $n$ of components $S_{i}$, which also coincides with $h^{1}\left(\mathcal{O}_{C}\right)$. The difference of vertices and edges is

$$
v-e=\chi(\Gamma(D))=h^{0}(\Gamma(D), k)-h^{1}(\Gamma(D), k)=1-h^{1}\left(\mathcal{O}_{D}\right) .
$$

Here we use $h^{1}(\Gamma(D), k)=h^{1}\left(\mathcal{O}_{D}\right)$, which follows from the exact sequence

$$
H^{0}\left(\tilde{D}, \mathcal{O}_{\tilde{D}}\right) \oplus H^{0}\left(D^{\prime}, \mathcal{O}_{D^{\prime}}\right) \longrightarrow H^{0}\left(\nu^{-1}\left(D^{\prime}\right), \mathcal{O}_{\nu^{-1}\left(D^{\prime}\right)}\right) \longrightarrow H^{1}\left(D, \mathcal{O}_{D}\right) \longrightarrow 0
$$

for the normalization $\nu: \tilde{D} \rightarrow D$, with $D^{\prime}=\operatorname{Sing}(D)$. The exact sequence

$$
0 \longrightarrow H^{1}\left(X, \mathcal{O}_{X}\right) \longrightarrow H^{1}\left(D, \mathcal{O}_{D}\right) \longrightarrow H^{1}\left(C, \mathcal{O}_{C}\right) \longrightarrow H^{2}\left(X, \mathcal{O}_{X}\right) \longrightarrow 0
$$

gives $h^{1}\left(\mathcal{O}_{D}\right)=h^{1}\left(\mathcal{O}_{C}\right)$. The upshot of this is

$$
\chi=(v-e)+f=1-h^{1}\left(\mathcal{O}_{D}\right)+h^{1}\left(\mathcal{O}_{C}\right)=1 .
$$

By the classification of compact real 2-manifolds, $\Pi(X)$ is a cell decomposition of the Klein bottle $\mathbb{R P}^{2}$.

Example 2.10. Let me discuss the easiest type III surfaces, which is nonsimple with $X=X_{1} \cup X_{2}$ two irreducible components. The following picture illustrates the gluing map $\varphi: C \rightarrow D$ :
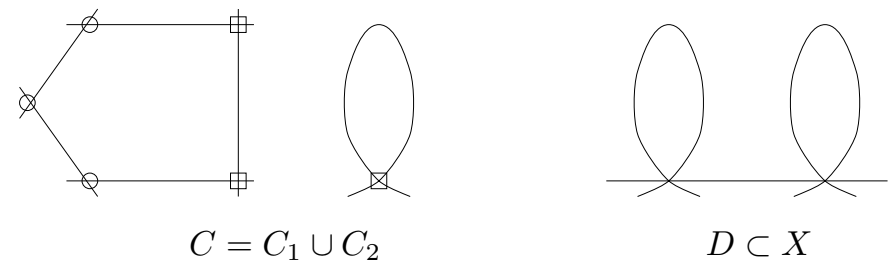

The symbols on the intersection points indicate the gluing. The component $S_{1} \subset S$ could be the blowing-up of a Hirzebruch surface $\bar{S}_{1}$ with an anticanonical 4-cycle of rational curves $\bar{C}_{1} \subset \bar{S}_{1}$ (two disjoint sections and two fibers), so that the center of the blowing-up is a singularity of $\bar{C}_{1}$. For $C_{2} \subset S_{2}$, choose a nodal cubic in $\mathbb{P}^{2}$. The cell decomposition $\Gamma(X)$ is given by an immersion of the 1-skeleton $\Gamma(D)$ :

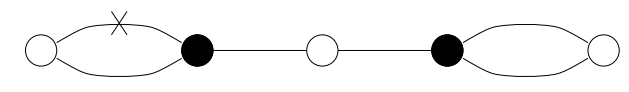

Here the unmarked edges have untwisted ribbons, whereas the marked edge has a twisted ribbon. There is another Enriques surfaces with two irreducible components, such that $C_{1}$ is a 4 -cycle, and $C_{2}$ is a 2 -cycle of rational curves. 
Remark 2.11. As in the smooth case, there is a canonical double covering for a normal crossing Enriques surfaces $X$ : According to Raynaud [23], Proposition 6.2.1, the inclusion $\operatorname{Pic}_{X}^{\tau} \subset \operatorname{Pic}_{X}$ corresponds to a nontrivial principal homogeneous $G$ space $r: \tilde{X} \rightarrow X$, where $G=\operatorname{Hom}\left(\operatorname{Pic}_{X}^{\tau}, \mathbb{G}_{m}\right)$ is the Cartier dual. The surface $\tilde{X}$ is locally of complete intersection and has $\Gamma\left(\tilde{X}, \mathcal{O}_{\tilde{X}}\right)=k$. Moreover, $H^{1}\left(\tilde{X}, \mathcal{O}_{\tilde{X}}\right)=0$ and $K_{\tilde{X}}=0$. This suggests to call $\tilde{X}$ the K3-like covering of $X$.

For ordinary Enriques surfaces, $G=\mathbb{Z} / 2 \mathbb{Z}$ is étale, so the K3-like covering $\tilde{X}$ is a normal crossing K3 surface. Suppose that $X$ is classical or supersingular. Then $G$ is radical, so the covering $r: \tilde{X} \rightarrow X$ is radical. With the notation of Theorem 2.7, you can easily see that the induced homogeneous $G$-space $r_{i}: \tilde{X}_{i} \rightarrow X_{i}$ is trivial for $i=1, \ldots n-1$. Note that the K3-like covering $\tilde{X}$ is nonreduced.

\section{Projectivity and D-Semistability}

Over the complex numbers, each smooth analytic Enriques surface is projective ([1], p. 184). Are normal crossing Enriques surface projective? In this section, we shall analyze this problem for type III surfaces $X$. Let $C_{j} \subset C$ be the irreducible components of the ramification curve $C \subset S$ on the normalization $S$. Following Miranda and Morrison [19], we say that $X$ is in minus-one-form if

$$
C_{j}^{2}=\left\{\begin{aligned}
-1 & \text { if } C_{j} \subset S \text { is smooth } \\
+1 & \text { if } C_{j} \subset S \text { is nodal, }
\end{aligned}\right.
$$

for all irreducible components $C_{j} \subset C$. Equivalently, $C \cdot C_{j}=1$ for every irreducible component $C_{j}$.

Proposition 3.1. Enriques surfaces of type III in minus-one-form are projective.

Proof. Let $X$ be such a surface. An invertible $\mathcal{O}_{S}$-module $\mathcal{L}$ descends to $X$ if and only if its restriction $\mathcal{L}_{C}$ lies in $\operatorname{Pic}(D) \subset \operatorname{Pic}(C)$. Of course, a necessary condition is that the numerical class of $\mathcal{L}_{C}$ lies in $\mathrm{NS}(D) \subset \mathrm{NS}(C)$. I claim that this is also sufficient: The exact sequence

$$
0 \longrightarrow H^{1}\left(X, \mathcal{O}_{X}\right) \longrightarrow H^{1}\left(D, \mathcal{O}_{D}\right) \longrightarrow H^{1}\left(C, \mathcal{O}_{C}\right) \longrightarrow H^{2}\left(X, \mathcal{O}_{X}\right) \longrightarrow 0
$$

shows that the Jacobians $\operatorname{Pic}_{D}^{0}$ and $\mathrm{Pic}_{C}^{0}$ have the same dimensions. Consequently, the map $\operatorname{Pic}_{D}^{0} \rightarrow \operatorname{Pic}_{C}^{0}$ must be surjective, because its kernel $\mu_{2}$ is infinitesimal. The claim follows.

The divisor $C$ is ample on itself, because $X$ is in minus-one-form. By the FujitaZariski Theorem (10], Thm. 1.10), the divisor $C$ is semiample. The corresponding birational contraction $S \rightarrow S^{\prime}$ has an exceptional curve $R \subset S$ disjoint from $C$. Choose an $S^{\prime}$-ample divisor $A \in \operatorname{Div}(S)$ supported by $R$. Then $t C+A$ is ample for $t \gg 0$. By construction, the class of $t C+A$ in $\mathrm{NS}(C)$ lies in the subgroup $\mathrm{NS}(D)$. Consequently, the ample invertible $\mathcal{O}_{S}$-module $\mathcal{O}_{S}(t C+A)$ descend to an ample invertible $\mathcal{O}_{X}$-module.

In light of this, we seek to put any type III surfaces into minus-one-form. To do so, I have to recall Kulikov's concept of type I and type II modifications [18. Let $E \subset D$ be smooth double curve with selfintersection -1 on each adjacent component. Blowing-up $E$ creates a 4 -cycle of $(-1)$-curves. Blowing-down the strict transform of $E$ yields another Enriques surface of type III called the modification of type II. The picture is: 


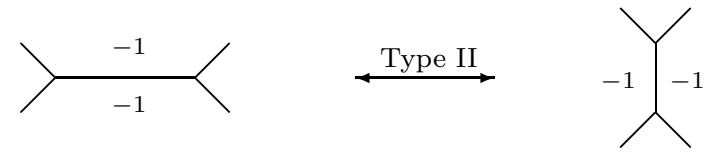

Now let $E \subset S$ be an exceptional curve of the first kind outside $C$ corresponding to the dotted lines below. Blowing-up $E$ and contracting its strict transform yields another Enriques surface of type III, called the modification of type I. Here the picture is:

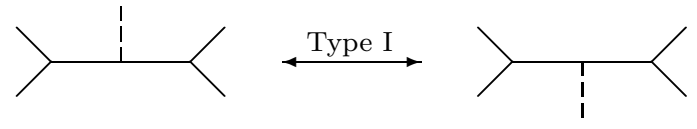

Recall that a normal crossing surface $X$ is called $d$-semistable if the the sheaf of first-order deformations

$$
\mathcal{T}_{X}^{1}=\mathcal{E} x t^{1}\left(\Omega_{X / k}^{1}, \mathcal{O}_{X}\right)
$$

is isomorphic to $\mathcal{O}_{D}$. Friedman [9] showed that d-semistability is necessary for the existence of smoothings with smooth total space.

Proposition 3.2. For each d-semistable Enriques surface of type III, there is a sequence of type I and type II modifications reaching a d-semistable Enriques surface of type III in minus-one-form.

Proof. Miranda and Morrison [19] proved this difficult result for Kulikov degenerations of K3 surfaces. Their arguments apply unchanged to our situation.

For later use, we record the following observation.

Lemma 3.3. Suppose $X$ is in minus-one-form. Then there are at least two components $S_{1}, S_{2}$ so that the irreducible components of $C$ contained in $S_{1} \cup S_{2}$ have linear independent Hodge classes in the $k$-vector space $H^{1,1}(S)=H^{1}\left(S, \Omega_{S / k}^{1}\right)$.

Proof. First, suppose that the irreducible components of $C \subset S$ are smooth. Let $m>0$ be the number of irreducible components in $C$. Then $D$ has $m / 2$ irreducible components and $m / 3$ singularities. Moreover, the normalization map $\tilde{D} \rightarrow D$ has $m$ ramification points. Consequently,

$$
1=\Pi(X)=m / 2+m / 3-m+n=n-m / 6 .
$$

For each integer $r>0$, let $n_{r}$ be the number of components $S_{i}$ so that $C \cap S_{i}$ is an $r$-cycle of rational curves. Then $n=\sum n_{r}$ and $m=\sum r n_{r}$, so $6=\sum n_{r}(6-r)$. We infer that at least two components, say $S_{1}, S_{2}$, have a ramification curve with $r \leq 5$ irreducible components. According to 19, Lemma 11.5, each irreducible component of $C$ in $S_{1} \cup S_{2}$ meets an exceptional curve of the first kind not contained in $C$. The assertion follows.

Second, suppose that there is a nodal components $C^{\prime} \subset C$, such that $\left(C^{\prime}\right)^{2}=1$. If there is another nodal component, we are done immediately, so let us assume that the other components are smooth. Then $D$ has $m / 2$ irreducible components and $(m-1) / 3$ singularities. Moreover, the normalization map $\tilde{D} \rightarrow D$ has $m-1$ ramification points. Now a calculation as above finishes the proof.

The K3-like covering of a type III Enriques surfaces is a K3 surface of type III. Let me remark that there is no analog of Proposition 3.1 for such surfaces: 
Proposition 3.4. K3 surfaces of type III with generic gluing map do not embed into smooth separated schemes.

Proof. Let $X$ be an arbitrary K3 surface type III. We have to show that $X$ does not embed into smooth schemes if the gluing map $\varphi: C \rightarrow D$ is generic. Here, as usual, $D$ is the singular locus, and $C \subset S$ the ramification locus. Decompose $D=D_{1} \cup \ldots \cup D_{m}$ into irreducible components. Let $k^{\times}$act on each $D_{i}$ fixing the triple points. This gives $\operatorname{Aut}^{0}(D)=\prod_{i=1}^{m} k^{\times}$. We can view $T=\operatorname{Aut}^{0}(D)$ as a parameter space for gluing morphisms $\varphi: C \rightarrow D$ in the following way. For each $D_{i}$, choose an ordering $\nu^{-1}\left(D_{i}\right)=C_{i}^{\prime} \cup C_{i}^{\prime \prime}$. Set $C^{\prime}=\cup C_{i}^{\prime}$ and $C^{\prime \prime}=\cup C_{i}^{\prime \prime}$. Then each $\lambda=\left(\lambda_{1}, \ldots, \lambda_{m}\right)$ in $T=\operatorname{Aut}^{0}(D)$ is also an automorphism $\tilde{\lambda}: C \rightarrow C$, defined by $\left.\tilde{\lambda}\right|_{C^{\prime}}=\lambda$ and $\tilde{\lambda} \mid C^{\prime \prime}=$ id. So each $\lambda \in T$ defines a new gluing morphism $\varphi_{\lambda}=\varphi \circ \tilde{\lambda}$. We obtain a locally trivial flat family $X_{\lambda}, \lambda \in T$ of type III surfaces.

How does the Picard group $\operatorname{Pic}\left(X_{\lambda}\right)$ jump in this family? An invertible $\mathcal{O}_{S^{-}}$ module $\mathcal{L}$ descends to $X$ if and only if its restriction $\mathcal{L}_{C}$ lies in $\operatorname{Pic}(D) \subset \operatorname{Pic}(C)$. Of course, a necessary condition is that the numerical class of $\mathcal{L}_{C}$ lies in $\operatorname{NS}(D) \subset$ $\mathrm{NS}(C)$, in other words, $\left(\mathcal{L} \cdot C_{i}^{\prime}\right)=\left(\mathcal{L} \cdot C_{i}^{\prime \prime}\right)$. Suppose this is the case and set $d_{i}=$ $\left(\mathcal{L} \cdot C_{i}^{\prime}\right)$. Let $\operatorname{Pic}^{d}(C) \subset \operatorname{Pic}(C)$ be the connected component with $\mathcal{L}_{C} \in \operatorname{Pic}^{d}(C)$ and $\operatorname{Pic}^{d}(D) \subset \operatorname{Pic}(D)$ the corresponding connected component. The exact sequence

$$
0 \longrightarrow H^{1}\left(D, \mathcal{O}_{D}\right) \longrightarrow H^{1}\left(C, \mathcal{O}_{C}\right) \longrightarrow H^{2}\left(X, \mathcal{O}_{X}\right) \longrightarrow 0
$$

and $h^{2}\left(X, \mathcal{O}_{X}\right)=1$ implies that there is an exact sequence

$$
0 \longrightarrow \operatorname{Pic}^{0}(D) \longrightarrow \operatorname{Pic}^{0}(C) \longrightarrow k^{\times} \longrightarrow 1 \text {. }
$$

Hence the inclusion $\operatorname{Pic}^{d}(D) \subset \operatorname{Pic}^{d}(C)$ has codimension 1. From this we infer that the set of all parameters $T_{\mathcal{L}} \subset T$ for which $\mathcal{L}$ descends to $X$ is either empty, or of codimension 1 , or equals $T$.

Suppose that $\mathcal{L}_{C}$ has at least one degree $d_{j} \neq 0$. Consider parameters $\lambda=$ $\left(\lambda_{1}, \ldots, \lambda_{m}\right)$ in $T=\prod_{i=1}^{m} k^{\times}$with $\lambda_{i}=1$ for $i \neq j$. You easily check that $\tilde{\lambda}^{*}\left(\mathcal{L}_{C}\right) \simeq$ $\mathcal{L}_{C}$ holds if and only if $\lambda_{j} \in k^{\times}$is a $d_{j}$-th root of unity. It follows that $T_{\mathcal{L}} \subset T$ is of codimension 1. Thus the set of all $\lambda \in T$ for which some $\mathcal{L} \in \operatorname{Pic}(S)$ with $\mathcal{L}_{C} \not \equiv \mathcal{O}_{C}$ descends to $X$ is a countable union of codimension 1 subsets. Consequently, no such $\mathcal{L}$ exists for $\lambda \in T$ generic.

Now we argue as follows: Seeking a contradiction, suppose that, for generic gluing map $\varphi: C \rightarrow D$, there is a closed embedding $X \subset Y$ into a smooth separated scheme $Y$. Choose an affine open neighborhood $U \subset Y$ intersecting $D$. Then $Y \backslash U$ defines a Cartier divisor on $Y$, whose restriction to $X$ is numerically nontrivial on $D$, contradiction.

Remark 3.5. Borelli [4] calls a scheme $Y$ is divisorial if the open subsets of the form $Y_{s} \subset Y$ generate the topology, where $s \in \Gamma(Y, \mathcal{L})$ ranges over sections of line bundles $\mathcal{L} \in \operatorname{Pic}(Y)$. The preceding proof shows that it is impossible to embed generic K3 surfaces of type III into divisorial schemes.

\section{Fibrations And D-Semistability}

Smooth Enriques surface carry elliptic or quasielliptic fibrations ([6], Thm. 5.7.1). Do semistable Enriques surface admit a genus one fibration? In this section we shall find partial answers for type II surfaces. First, we observe that there is always an ample line bundle: 
Proposition 4.1. Enriques surface of type II are projective.

Proof. Let $X$ be such a surface. To construct an ample invertible $\mathcal{O}_{X}$-module, it suffices to find an ample invertible $\mathcal{O}_{S}$-module $\mathcal{L}$ whose restriction $\mathcal{L}_{C}$ lies in the image of $\operatorname{Pic}(D)$. Let us do the case that $X$ is nonsimple and reducible (the other cases are similar). We use the notation from Theorem 2.5. Start with the rational component $S_{1}$ and choose an ample invertible $\mathcal{O}_{S_{1}}$-module $\mathcal{L}_{1}$. Next, choose an ample invertible $\mathcal{O}_{S_{2}}$-module $\mathcal{L}_{2}$. Passing to suitable multiples if necessary, we can assume that $\left(\mathcal{L}_{1} \cdot C_{1}\right)=\left(\mathcal{L}_{2} \cdot C_{2}^{\prime \prime}\right)$, where $C_{2}^{\prime \prime} \subset C_{2}$ is the connected component with $\varphi\left(C_{1}\right)=\varphi\left(C_{2}^{\prime \prime}\right)$. Since $\operatorname{Pic}^{0}\left(S_{2}\right) \rightarrow \operatorname{Pic}^{0}\left(C_{2}^{\prime \prime}\right)$ is surjective, we can modify $\mathcal{L}_{2}$ by a numerically trivial sheaf so that $\left.\left.\mathcal{L}_{1}\right|_{C_{1}} \simeq \mathcal{L}_{2}\right|_{C_{2}^{\prime \prime}}$ with respect to the gluing morphisms.

Now proceed by induction: This gives a sequence $\mathcal{L}_{1}, \ldots, \mathcal{L}_{n}$ of ample invertible $\mathcal{O}_{S_{i}}$-modules which coincides on the overlaps. Let $D_{n} \subset D$ be the image of the last ramification curve $C_{n}^{\prime} \subset C_{n}$. Then $C_{n}^{\prime} \rightarrow D_{n}$ is a $\mathbb{Z} / 2 \mathbb{Z}$ double covering, such that $\operatorname{Pic}\left(D_{n}\right) \rightarrow \operatorname{Pic}\left(C_{n}^{\prime}\right)$ has a cokernel $\mathbb{Z} / 2 \mathbb{Z}$. Replacing the $\mathcal{L}=\mathcal{L}_{1} \cup \ldots \mathcal{L}_{n}$ by a suitable multiple if necessary, we can assume that $\mathcal{L}$ is an invertible $\mathcal{O}_{S}$-module whose restriction $\mathcal{L}_{C}$ lies in the image of $\operatorname{Pic}(D)$.

In the following, $X$ will be a Enriques surface of type II. Then the sheaf $\mathcal{T}_{X}^{1}=$ $\mathcal{E}_{x} t^{1}\left(\Omega_{X / k}, \mathcal{O}_{X}\right)$ is dual to the norm $N_{C / D}(\mathcal{N})$, where $\mathcal{N}=\mathcal{N}_{C / S}$ is the conormal bundle. Let me state the following fact:

Lemma 4.2. Suppose $X$ is an Enriques surface of type II. Let $D^{\prime} \subset D$ be a connected component with connected preimage $C^{\prime} \subset C$. Then $\left.\mathcal{T}_{X}^{1}\right|_{D^{\prime}} \simeq \mathcal{O}_{D^{\prime}}$ holds if and only if the conormal bundle $\mathcal{N}_{C^{\prime} / S} \in \operatorname{Pic}\left(C^{\prime}\right)$ is 2-torsion.

Proof. Set $\mathcal{N}=\mathcal{N}_{C^{\prime} / S}$. We have an exact sequence

$$
0 \longrightarrow D(\mathbb{Z} / 2 \mathbb{Z}) \longrightarrow \mathrm{Pic}_{D^{\prime}}^{0} \longrightarrow \mathrm{Pic}_{C^{\prime}}^{0} \longrightarrow 0,
$$

so $\operatorname{Pic}^{0}\left(D^{\prime}\right) \rightarrow \operatorname{Pic}^{0}\left(C^{\prime}\right)$ is bijective. Suppose $\mathcal{N} \otimes 2 \simeq \mathcal{O}_{C^{\prime}}$. Choose an invertible $\mathcal{O}_{D^{\prime}}$-module $\mathcal{L}$ with $\mathcal{N}=\nu^{*}(\mathcal{L})$. Then $\mathrm{N}_{C^{\prime} / D^{\prime}}(\mathcal{N})=\mathcal{L}^{\otimes 2}$ is trivial, so $\left.\mathcal{T}_{X}^{1}\right|_{D^{\prime}} \simeq \mathcal{O}_{D^{\prime}}$ The converse is similar.

The condition of d-semistability does not restrict the number of irreducible components of $X$. Rather, it says something about the minimal model of the normalization:

Proposition 4.3. Suppose $X$ is d-semistable and of type II. Let $\bar{S}$ be a minimal model of $S$. Then the corresponding contraction $h: S \rightarrow \bar{S}$ is a sequence of 8 or 9 blowing-ups.

Proof. The number $m \geq 0$ of blowing-ups in $h: S \rightarrow \bar{S}$ is given by $K_{S}^{2}=K_{\bar{S}}^{2}-m$. On the other hand, d-semistability implies $K_{S}^{2}=C^{2}=0$. According to Theorem 2.5 and Theorem 2.7, there is precisely one rational components $\bar{S}_{1} \subset \bar{S}$, which has $K_{\bar{S}_{1}}^{2}=8$ or $K_{\bar{S}_{1}}^{2}=9$. Moreover, the other minimal models have $K_{\bar{S}_{i}}^{2}=0$. This proves the assertion.

Regular but nonsmooth curves of arithmetic genus one are called quasielliptic. They do not exist over algebraically closed fields, but may occur over function fields:

Proposition 4.4. Suppose that $X$ is a nonsimple d-semistable Enriques surface of type II. Then there is a proper morphism $g: X \rightarrow \mathbb{P}^{1}$ with $\mathcal{O}_{\mathbb{P}^{1}} \rightarrow g_{*}\left(\mathcal{O}_{X}\right)$ bijective, so that the generic fiber $X_{\eta}$ is an elliptic or quasielliptic curve. 
Proof. First, assume that $X$ is irreducible. According to Theorem 2.5, the normalization $S$ is rational, and the ramification locus $C \subset S$ is an elliptic curve. By Lemma 4.2, the conormal sheaf $\mathcal{N}=\mathcal{O}_{C}(-C)$ is 2-torsion. Assume that $\mathcal{N}$ is trivial. Then the exact sequence

$$
0 \longrightarrow \mathcal{O}_{X} \longrightarrow \mathcal{O}_{X}(C) \longrightarrow \mathcal{O}_{C} \longrightarrow 0
$$

yields an exact sequence

$$
0 \longrightarrow H^{0}\left(S, \mathcal{O}_{S}\right) \longrightarrow H^{0}\left(S, \mathcal{O}_{S}(C)\right) \longrightarrow H^{0}\left(C, \mathcal{O}_{C}\right) \longrightarrow 0,
$$

hence $C$ is base-point-free and defines the desired fibration. Now assume that $\mathcal{N}$ has order 2. The exact sequence

$$
0 \longrightarrow \mathcal{O}_{S} \longrightarrow \mathcal{O}_{S}(2 C) \longrightarrow \mathcal{O}_{2 C}(2 C) \longrightarrow 0
$$

yields an exact sequence

$$
0 \longrightarrow H^{0}\left(S, \mathcal{O}_{S}\right) \longrightarrow H^{0}\left(S, \mathcal{O}_{S}(2 C)\right) \longrightarrow H^{0}\left(2 C, \mathcal{O}_{2 C}(2 C)\right) \longrightarrow 0 .
$$

Moreover, the exact sequence

$$
0 \longrightarrow O_{C}(C) \longrightarrow \mathcal{O}_{2 C}(2 C) \longrightarrow \mathcal{O}_{C} \longrightarrow 0
$$

implies that $H^{0}\left(\mathcal{O}_{2 C}(2 C)\right) \rightarrow H^{0}\left(\mathcal{O}_{C}\right)$ is bijective, hence $2 C$ is base-point-free. In both cases we obtain a genus one fibration $S \rightarrow \mathbb{P}^{1}$, which clearly descends to a genus one fibration $g: X \rightarrow \mathbb{P}^{1}$.

Next, assume that $X$ is not irreducible. Using the notation from Theorem 2.5, we shall consider the last component $S_{n} \subset S$ and its ruling $f_{n}: S_{n} \rightarrow B_{n}$. Let $C^{\prime} \subset C_{n}$ be the connected component double covering its image $D^{\prime} \subset D$. By d-semistability, $\mathcal{N}=\mathcal{N}_{C^{\prime} / S}$ is 2 -torsion. Setting $\mathcal{E}=\left(f_{n}\right)_{*}\left(\mathcal{O}_{S_{n}}\left(C^{\prime}\right)\right)$ and $\mathcal{L}=\left(f_{n}\right)_{*}(\mathcal{N})$, we obtain an exact sequence

$$
0 \longrightarrow \mathcal{O}_{B_{n}} \longrightarrow \mathcal{E} \longrightarrow \mathcal{L} \longrightarrow 0 \text {. }
$$

Note that $\bar{S}_{n}=\mathbb{P}(\mathcal{E})$ gives a minimal model $h: S_{n} \rightarrow \bar{S}_{n}$. Suppose that $\mathcal{N}$ is trivial. Since the other ramification curve $C^{\prime \prime} \subset C_{n}$ has image $h\left(C_{n}^{\prime \prime}\right)$ disjoint from $h\left(C^{\prime}\right)$, the preceding extension splits, and $\bar{S}_{n}=B_{n} \times \mathbb{P}^{1}$. So we obtain an elliptic structure $S_{n} \rightarrow \mathbb{P}^{1}$.

Now suppose that $\mathcal{N}$ has order 2. The corresponding inclusion $\mathbb{Z} / 2 \mathbb{Z} \subset \operatorname{Pic}_{B_{n}}$ defines a principle homogeneous $\mu_{2}$-space over $B_{n}$ on which $\mathcal{N}$ becomes trivial. It follows that $\operatorname{Fr}^{*}(\mathcal{N})$ is trivial. We infer that there is an elliptic structure on $\mathbb{P}\left(\operatorname{Fr}^{*}(\mathcal{E})\right)$ which descends to a genus one fibration on $\bar{S}_{n}=\mathbb{P}(\mathcal{E})$. In both cases, the fibrations induce a morphism $X \rightarrow \mathbb{P}^{1}$. Note that the initial components $S_{1}, \ldots, S_{n-1}$ are mapped to points.

Here is another kind of fibration. Suppose that $X$ is of type II as described in Theorem 2.5 or 2.7. Additionally, assume that the rational component $S_{1} \subset S$ is not $\mathbb{P}^{2}$. Choose a ruling $f_{1}: S_{1} \rightarrow \mathbb{P}^{1}$. Together with the elliptic rulings $f_{i}: S_{i} \rightarrow B_{i}$ for $2 \leq i \leq n$, this defines a ruling $f: S \rightarrow B$ over $B=\mathbb{P}^{1} \cup B_{2} \cup \ldots \cup B_{n}$. We seek to descend this fibration on $S$ to a fibration on $X$ mapping to a finite quotient of the curve $B$.

Proposition 4.5. With the preceding assumptions, the fibration $f: S \rightarrow B$ descends to a fibration $g: X \rightarrow \mathbb{P}^{1}$. The generic fiber is a curve (possibly nonreduced) of arithmetic genus $p_{a}\left(X_{\eta}\right)=1$. 
Proof. Let $E$ be the elliptic curve isomorphic to the components of $C$. The induced projection $f_{1}: C_{1} \rightarrow \mathbb{P}^{1}$ defines an involution $\iota: E \rightarrow E$, hence a subgroup $\mathbb{Z} / 2 \mathbb{Z} \subset \operatorname{Aut}(E)$. There is another subgroup scheme $H \subset \operatorname{Aut}(E)$ of length two: If $X$ is simple, we define $H$ by the double covering $C_{n} \rightarrow B_{n}$; if $X$ is nonsimple, $H=\mathbb{Z} / 2 \mathbb{Z}$ is defined by the part of the gluing morphism that is responsible for the nonsmooth component $X_{n}$. Let $G \subset \operatorname{Aut}(E)$ be the subgroup scheme of length 4 generated by $H$ and the involution $\iota: E \rightarrow E$. Then $E / G \simeq \mathbb{P}^{1}$. It is easy to see that $f: S \rightarrow B$ induces a fibration $g: X \rightarrow E / G$.

It remains to determine the generic fiber. First, suppose that $X$ is nonsimple. Then $X_{\eta}$ is a cycle of rational curves with $2+4(n-1)=4 n-2$ irreducible components. Here each elliptic ruled component $S_{i}$ contains 4 irreducible components of $X_{\eta}$. Second, suppose that $X$ is simple. Then $X_{\eta}$ is nonreduced: it is a string of the form $X_{\eta}=\mathbb{P}^{1}+2 \mathbb{P}^{1}+\ldots+2 \mathbb{P}^{1}+\mathbb{P}^{1}$ with $1+2(n-1)=2 n-1$ irreducible components. Here the reduced part $\mathbb{P}^{1}+\mathbb{P}^{1}$ lies on the last irreducible component $X_{n} \subset X$. From this, you immediately get $p_{a}\left(X_{\eta}\right)=1$.

\section{USING LOG STRUCTURES TO CONSTRUCT FORMAL SMOOTHINGS}

In this section, the task is to find formal deformations of d-semistable Enriques surfaces. This will be a major step towards the construction of algebraic deformations, which eventually leads to smoothings and liftings. A direct approach would be to study a versal deformation $\mathfrak{X} \rightarrow \operatorname{Spf}(R)$. The problem, however, is that $X=\mathfrak{X}_{0}$ has many locally trivial deformations, which are irrelevant for our purposes, and this fact obscures the structure of $\operatorname{Spec}(R)$.

A natural way to avoid such problems is to use logarithmic structures. The underlying idea is to enlarge the category of schemes so that d-semistable schemes can be considered as smooth schemes. Let my recall the fundamental definition.

Definition 5.1. (Kato) A $\log$ scheme $Y^{\dagger}=\left(Y, \mathcal{M}_{Y}, \alpha\right)$ comprises a scheme $Y$, a sheaf of monoids $\mathcal{M}_{Y}$, and a homomorphism $\alpha: \mathcal{M}_{Y} \rightarrow \mathcal{O}_{Y}$ so that the map $\alpha^{-1}\left(\mathcal{O}_{X}^{\times}\right) \rightarrow \mathcal{O}_{X}^{\times}$is bijective.

Here $\mathcal{M}_{Y}$ is a sheaf in the étale topology, and $\alpha: \mathcal{M}_{Y} \rightarrow \mathcal{O}_{Y}$ is a homomorphism with respect to multiplication in $\mathcal{O}_{X}$. You find more about log structures in K. Kato's fundamental paper [16] and Illusie's survey article 14]. Suppose $X$ is a d-semistable normal crossing surface. We shall use log structures on $X$, on its normalization $S$, and on the ground field $k$.

Let us start with the ground field. Set $\mathcal{M}_{k}=\mathbb{N} \oplus k^{\times}$. The function $\alpha: \mathcal{M} \rightarrow k$ with $\alpha(0, \lambda)=\lambda$ and $\alpha(n, \lambda)=0$ for $n \neq 0$ defines a log structure $k^{\dagger}$ called the standard log structure. The corresponding $\log \operatorname{scheme} \operatorname{Spec}\left(k^{\dagger}\right)$ is nicknamed the 'punctured point'.

Next, consider the normalization $S$ of $X$. Let $j: U \rightarrow S$ be the complement of the ramification locus $C \subset S$ for normalization. Set $\mathcal{M}_{S}=\mathcal{O}_{S} \cap j^{*}\left(\mathcal{O}_{U}^{\times}\right)$. The canonical map $\alpha: \mathcal{M}_{S} \rightarrow \mathcal{O}_{S}$ yields a $\log$ structure $S^{\dagger}$.

Finally, we come to the normal crossing surface $X$. Locally, there is a closed embedding $i: X \rightarrow \mathbb{A}^{3}$ as a union of coordinate hyperplanes. Pulling back the log structure $\mathcal{O}_{\mathbb{A}^{3}} \cap \mathcal{O}_{\mathbb{A}^{3} \backslash X}^{\times}$defines local log structures on $X$. Of course, these local log structures might not be compatible. However, F. Kato [15], Theorem 11.7 showed that a compatible choice is possible if $X$ is d-semistable. The corresponding global $\log$ structures are called of semistable type. Fix such a log structure of semistable 
type $X^{\dagger}$. By 15], Example 4.7, the structure morphism induces a log smooth morphism $X^{\dagger} \rightarrow \operatorname{Spec}\left(k^{\dagger}\right)$. This means that the lifting criterion for smoothness holds in the category of log schemes.

We seek to extend log structures of semistable type $X^{\dagger}$ over local Artin rings. Let $W$ be a complete discrete valuation ring of mixed characteristic with residue field $k$. (Choose $W \subset W(k)$ as the Cohen subring of the ring of Witt vectors, as in Bourbaki [5], Chap. IX.)

To keep track of the log structures, we introduce a formal variable $T$. The power series ring $W[[T]]^{\dagger}$ is endowed with the $\log$ structure given by $\mathbb{N} \rightarrow W[[T]], n \mapsto T^{n}$. Let $\left(\operatorname{Art}_{W[[T]]}\right)$ be the category of Artin local $W[[T]]$-algebras with residue field $k$. Each such Artin ring $A$ inherits a $\log$ structure $A^{\dagger}$ from the log structure $W[[T]]^{\dagger}$. For example, the residue field $k$ is endowed with the standard log structure.

Let $\operatorname{LD}_{X^{\dagger}}(A)$ be the set, modulo isomorphism, of $\log$ smooth morphisms $Y^{\dagger} \rightarrow$ $\operatorname{Spec}\left(A^{\dagger}\right)$ extending the $\log$ smooth morphism $X^{\dagger} \rightarrow \operatorname{Spec}\left(k^{\dagger}\right)$. We call the corresponding functor $\mathrm{LD}_{X^{\dagger}}:\left(\operatorname{Art}_{W[T]]}\right) \rightarrow$ (Set) the log deformation functor of $X^{\dagger}$. The following is the main technical results of this paper:

Theorem 5.2. Suppose $X$ is a nonsmooth d-semistable Enriques surface. If $X$ is classical or ordinary, then the log deformation functor $\mathrm{LD}_{X^{\dagger}}:\left(\operatorname{Art}_{W[[T]]}\right) \rightarrow($ Set) is formally smooth.

Proof. Formal smoothness of $\mathrm{LD}_{X^{\dagger}}$ means: For each $\log$ smooth deformation $Y^{\dagger} / A^{\dagger}$ and each extension $B \rightarrow A$ with square-zero ideal $I \subset B$, it is possible to extend $Y^{\dagger}$ over $B^{\dagger}$. According to F. Kato [15], Proposition 8.6, the obstruction lies in $H^{2}\left(X, \mathcal{H o m}\left(\Omega_{X^{\dagger} / k^{\dagger}}^{1}, \mathcal{O}_{X}\right)\right) \otimes_{A} I$. Here $\Omega_{X^{\dagger} / k^{\dagger}}^{1}$ is the sheaf of log differentials (see 15, sect. 5), which is locally free. It suffices to check $H^{0}\left(X, \Omega_{X^{\dagger} / k^{\dagger}}^{1} \otimes \omega_{X}\right)=0$.

Do proceed we have to relate various differentials. Luckily, this was done in Friedman's paper [9], where the sheaf $\Omega_{X^{\dagger} / k^{\dagger}}^{1}$ appears under the name $\Lambda_{X}^{1}$. He constructed a commutative diagram of $\mathcal{O}_{X}$-modules

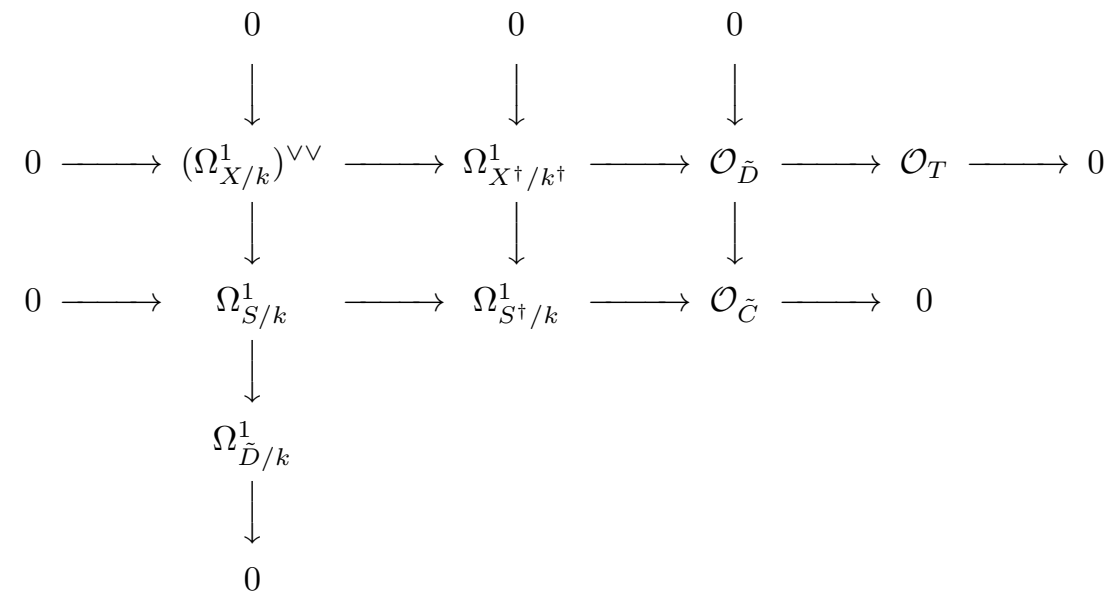

with exact rows and columns. Here $\mathcal{O}_{\tilde{D}} \rightarrow \mathcal{O}_{D}$ and $\mathcal{O}_{\tilde{C}} \rightarrow \mathcal{O}_{C}$ are the normalizations, and $T \subset X$ is the set of triple points. The second row displays $\Omega_{S^{\dagger} / k}^{1}$ as an inverse elementary transformation of $\Omega_{S / k}^{1}$. The map $\Omega_{S / k}^{1} \rightarrow \Omega_{\tilde{D} / k}^{1}$ is a Čech boundary operator explained in [9], p. 77. We shall treat three cases. 
(i) The case that $X$ is ordinary and of type II. There are no triple points, so $D=\tilde{D}$ and $C=\tilde{C}$. The upper row of Diagram (3) gives an exact sequence

$$
H^{0}\left(X,\left(\Omega_{X / k}^{1}\right)^{\vee \vee}\right) \longrightarrow H^{0}\left(X, \Omega_{X^{\dagger} / k^{\dagger}}^{1}\right) \longrightarrow H^{0}\left(D, \mathcal{O}_{D}\right) \longrightarrow H^{1}\left(X,\left(\Omega_{X / k}^{1}\right)^{\vee \vee}\right) .
$$

First, I claim that $H^{0}\left(X,\left(\Omega_{X / k}^{1}\right)^{\vee \vee}\right)=0$. Indeed: The left column of Diagram (3) gives an exact sequence

$$
0 \longrightarrow H^{0}\left(X,\left(\Omega_{X / k}^{1}\right)^{\vee \vee}\right) \longrightarrow H^{0}\left(S, \Omega_{S}^{1}\right) \longrightarrow H^{0}\left(D, \Omega_{D}^{1}\right)
$$

Using the notation from Theorem 2.7, we have $H^{1,0}\left(S_{1}\right)=0$ because the component $S_{1}$ is rational. Moreover, for $2 \leq i \leq n$, the maps in

$$
H^{1,0}\left(B_{i}\right) \longrightarrow H^{1,0}\left(S_{i}\right) \longrightarrow H^{1,0}\left(C \cap S_{i}\right)
$$

are injective because the projections $C \cap S_{i} \rightarrow B_{i}$ are étale. You easily infer that $H^{1,0}(S) \rightarrow H^{1,0}(D)$ is injective, so $H^{0}\left(X,\left(\Omega_{X / k}^{1}\right)^{\vee \vee}\right)=0$.

Second, I claim that the boundary map $H^{0}\left(\mathcal{O}_{D}\right) \rightarrow H^{1}\left(X,\left(\Omega_{X / k}^{1}\right)^{\vee \vee}\right)$ is injective. To see this, consider the commutative diagram

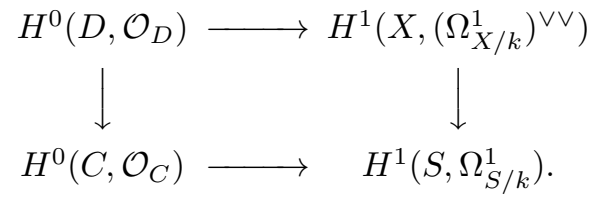

It suffices to check that the composition $H^{0}\left(D, \mathcal{O}_{D}\right) \rightarrow H^{1,1}(S)$ is injective. A direct manipulation with cocycles shows that the boundary map $H^{0}\left(C, \mathcal{O}_{C}\right) \rightarrow H^{1,1}(S)$ maps a section of $\mathcal{O}_{C}$ to the Hodge class of its support. Recall that the Hodgeclass-map $\operatorname{Pic}(S) \rightarrow H^{1,1}(S)$ is induced by logarithmic derivation

$$
\operatorname{dlog}: \mathcal{O}_{S}^{\times} \longrightarrow \Omega_{X}^{1}, \quad s \mapsto \operatorname{dlog}(s)=d s / s .
$$

Let $C_{j} \subset C$ be the irreducible components, and assume that some divisor $\sum \lambda_{j} C_{j}$ coming from $H^{0}(D, \mathcal{F})$ has zero Hodge class. By Proposition 4.3, there is an exceptional curve of the first kind $E \subset S$, say with $E \cdot C_{j}=1$. Then $\lambda_{j}=0$. Let $C_{k}$ be another component. If $\varphi\left(C_{k}\right)=\varphi\left(C_{j}\right)$ is a double curve, then $\lambda_{k}=\lambda_{j}$ is zero as well. If $C_{j}, C_{k}$ lie in the same ruled component $S_{i}$, the ruling $S_{i} \rightarrow B_{i}$ implies that $\lambda_{k}=\lambda_{j}=0$. Inductively, we conclude that all multiplicities in $\sum \lambda_{j} C_{j}$ vanish. It follows that $H^{0}\left(X, \Omega_{X^{\dagger} / k^{\dagger}}^{1}\right)=0$, and we conclude that the log deformation functor is formally smooth.

(ii) The case that $X$ is ordinary of type III. According to Theorem 2.9, the components $S_{i}$ are rational surface, so both $H^{0}\left(X,\left(\Omega_{X}^{1}\right)^{\vee \vee}\right) \subset H^{1,0}(S)$ must vanish. Consider the commutative diagram

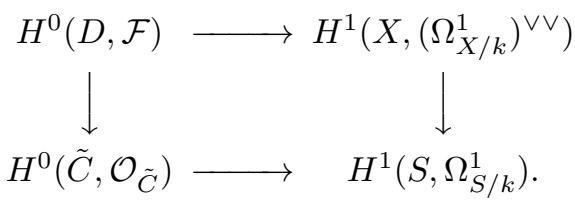

with $\mathcal{F}=\Omega_{X^{\dagger} / k^{\dagger}}^{1} /\left(\Omega_{X / k}^{1}\right)^{\vee \vee}$. We have to check that $H^{0}(D, \mathcal{F}) \rightarrow H^{1}\left(X,\left(\Omega_{X / k}^{1}\right)^{\vee \vee}\right)$ is injective. It suffices to show that the composition $H^{0}(D, \mathcal{F}) \rightarrow H^{1,1}(S)$ is injective. Let $C_{j} \subset C$ be the irreducible components and consider a nonzero divisor $\sum \lambda_{j} C_{j}$ coming from $H^{0}(D, \mathcal{F})$. This means that $\lambda_{j}=\lambda_{k}$ if the intersection $\varphi\left(C_{j}\right) \cap \varphi\left(C_{k}\right)$ is a double curve, and $\lambda_{j}+\lambda_{k}=\lambda_{l}$ if the intersection $\varphi\left(C_{j}\right) \cap \varphi\left(C_{k}\right) \cap \varphi\left(C_{l}\right)$ is a triple point. 
Seeking a contradiction, we assume that $\sum \lambda_{j} C_{j}$ has zero Hodge class. By Proposition 3.2, suitable modifications of type I and type II put $X$ into minus-oneform $X^{\prime}$. Let $C^{\prime} \subset S^{\prime}$ be the corresponding ramification curve, and $C_{j}^{\prime} \subset C^{\prime}$ be its irreducible components. The modifications give a canonical bijection between the $C_{j}$ and the $C_{j}^{\prime}$, and the nonzero divisor $\sum \lambda_{j} C_{j}^{\prime}$ has zero Hodge class in $H^{1,1}\left(S^{\prime}\right)$. According to Proposition 3.3, there is a component $S_{0}^{\prime} \subset S^{\prime}$ with $\lambda_{j}=0$ for all $C_{j}^{\prime} \subset S_{0}^{\prime}$. By the triple point condition, the multiplicities near $S_{0}^{\prime}$ look like:

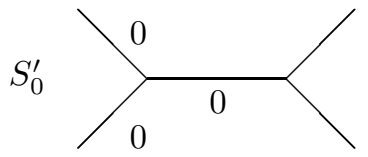

Using that $\sum \lambda_{j} C_{j}^{\prime}$ has zero Hodge class and that $X^{\prime}$ is in minus-one-form, we inductively infer that $\lambda_{j}=0$ for all $C_{j}$, contradiction. Again the log deformation functor is formally smooth.

(iii) The case that $X$ is classical. For the sake of simplicity, I only do the case that $X=X_{1} \cup X_{2}$ has $n=2$ irreducible components. By Theorem 2.7, the double curve $D=X_{1} \cap X_{2}$ is irreducible and $C$ comprises two irreducible components $C_{i} \subset S_{i}$. By Proposition 2.3, we have $\left.\nu^{*}\left(K_{X}\right)\right|_{S_{1}}=0$, whereas $\left.\nu^{*}\left(K_{X}\right)\right|_{S_{2}}$ has order two. You easily deduce that both $H^{0}\left(X,\left(\Omega_{X}^{1}\right)^{\vee \vee} \otimes \omega_{X}\right) \subset H^{0}\left(S, \Omega_{S / k}^{1} \otimes \omega_{X}\right)$ vanish.

It remains to check that the boundary map $H^{0}\left(\mathcal{O}_{D}\right) \rightarrow H^{1}\left(X,\left(\Omega_{X / k}^{1}\right)^{\vee \vee} \otimes \omega_{X}\right)$ is injective. Seeking a contradiction, we suppose that this map is zero. Then the map $H^{1}\left(X,\left(\Omega_{X / k}^{1}\right)^{\vee \vee} \otimes \omega_{X}\right) \rightarrow H^{1}\left(X, \Omega_{X^{\dagger} / k^{\dagger}}^{1} \otimes \omega_{X}\right)$ is injective, so the dual map

$$
H^{1}\left(X, \mathcal{H o m}\left(\Omega_{X^{\dagger} / k \dagger}^{1}, \mathcal{O}_{X}\right)\right) \longrightarrow H^{1}\left(X, \mathcal{H o m}\left(\Omega_{X / k}^{1}, \mathcal{O}_{X}\right)\right)
$$

is surjective. The group on the left is nothing but the tangential space $\operatorname{LD}_{X^{\dagger}}(k[\epsilon])$ for all locally trivial log deformations. The group on the right is the tangential space $H^{1}\left(X, \Theta_{X}\right)$ for all locally trivial deformations. The preceding surjection means that each first-order locally trivial deformation can be endowed with a log structure.

But this is absurd: By Proposition 4.3, there is an exceptional curve of the first kind $E \subset S$. Let $h: S \rightarrow \bar{S}$ be its contraction. Moving the center $\bar{s}=h(E)$ in $h(C) \subset \bar{S}$ over the dual numbers $k[\epsilon]$ destroys d-semistability for the corresponding first-order deformation of $X$. This gives an element in $H^{1}\left(X, \Theta_{X}\right)$ not in the image of $\operatorname{LD}_{X^{\dagger}}(k[\epsilon])$, contradiction. Again, we conclude that the log deformation functor is formally smooth.

\section{Supersingularity AND OBSTRUCTIONS}

In contrast to the classical and ordinary case, there are obstructions for supersingular Enriques surfaces.

Theorem 6.1. The log deformation functor $\mathrm{LD}_{X^{\dagger}}:\left(\operatorname{Art}_{W[[T]]}\right) \rightarrow($ Set $)$ of a nonsmooth d-semistable supersingular Enriques surface is not formally smooth.

This needs some preparations. First, let me recall the classification of group schemes of order two over an arbitrary ground ring $A$ of characteristic $p=2$. Fix two elements $a, b \in A$ with $a b=0$. Set

$$
\Lambda_{a}=A\left[T \mid T^{2}-a T\right] \quad \text { and } \quad \mu_{b}(T)=T \otimes 1+1 \otimes T+b T \otimes T .
$$


Then $G_{a, b}=\operatorname{Spec}\left(\Lambda_{a}\right)$ is a commutative group scheme of length two, with group law defined by $\mu_{b}: \Lambda_{a} \rightarrow \Lambda_{a} \otimes \Lambda_{a}$. The corresponding group-valued functor on the category of $A$-algebras is $G_{a, b}(R)=\left\{r \in R \mid r^{2}=a r\right\}$ with group law

$$
r_{1} * r_{2}=r_{1}+r_{2}+b r_{1} r_{2} \text {. }
$$

For example, $G_{0,0}=\alpha_{2}$, and $G_{1,0}=\mathbb{Z} / 2 \mathbb{Z}$, and $G_{0,1}=\mu_{2}$. There is an isomorphism $G_{a, b} \simeq G_{a^{\prime}, b^{\prime}}$ if and only if there is a unit $\gamma \in A^{\times}$with $a^{\prime}=\gamma a$ and $b^{\prime}=\gamma^{-1} b$. Swapping the indices gives Cartier duality: $D\left(G_{a, b}\right)=G_{b, a}$. Note that the augmentation ideal $T \Lambda \subset \Lambda$ is a free $A$-module of rank 1 .

Lemma 6.2. Let $G=\operatorname{Spec}(\Lambda)$ be a commutative affine group A-scheme whose augmentation ideal is a free $A$-module of rank 1. Then $G$ is isomorphic to $G_{a, b}$ for some $a, b \in A$ with $a b=0$.

This is a straightforward calculation. You find it in 25, Example 3.2. We shall apply this result in the following situation. Set $A=k[[t]]$, and let $\mathcal{E} \subset \mathbb{P}_{A}^{2}$ be the relative elliptic curve defined by the Weierstrass equation in Deuring normal form $y^{2}+t^{2} x y+y=x^{3}$. The closed fiber has $j\left(\mathcal{E}_{\sigma}\right)=0$, whereas $j\left(\mathcal{E}_{\eta}\right)=t^{33} /\left(t^{6}-1\right)$. Let $\mathcal{G}$ be the kernel over $A$ of the homomorphism $2: \mathcal{E} \rightarrow \mathcal{E}$.

Lemma 6.3. The group A-scheme $\mathcal{G}$ is isomorphic to $G_{t, 0}$.

Proof. The closed fiber is $\mathcal{G}_{\sigma}=G_{0,0}$. Using the group law on the generic fiber [24], p. 58 , you see that $\mathcal{G}_{\eta}=\mathbb{Z} / 2 \mathbb{Z}$, generated by the rational point $\left(t^{-2}, t^{-3}\right) \in \mathcal{E}(\eta)$. (This is why we use the coefficient $t^{2}$ instead of $t$ in the Weierstrass equation. Otherwise $2: \mathcal{E} \rightarrow \mathcal{E}$ would be a nontrivial $\mathcal{G}$-torsors.) According to Lemma 6.2, we have $\mathcal{G}=G_{t^{m}, 0}$ for some integer $m \geq 1$. Note that $m$ depends only on the underlying scheme structure of $\mathcal{G}$; in fact, it is the order of contact for the closures of the two points in $\mathcal{G}(\eta)$. The point $\left(t^{-2}, t^{-3}\right) \in \mathcal{G}(\eta)$ has homogeneous coordinates $\left(t: 1: t^{3}\right) 1152$, and we conclude $m=1$.

Next, we use the flat family $\mathcal{E} \rightarrow \operatorname{Spec}(A)$ of elliptic curves to construct flat families of type II Enriques surfaces.

Lemma 6.4. Suppose $X$ is a supersingular d-semistable Enriques surface of type II. Then there are two projective A-deformation $\mathfrak{X}_{1}, \mathfrak{X}_{2}$ of $X$ so that $\mathfrak{X}_{2} \otimes \kappa(\eta)$ is an ordinary d-semistable Enriques surface of type II, whereas $\mathfrak{X}_{1} \otimes \kappa(\eta)$ is a classical $d$-semistable Enriques surface of type II .

Proof. We shall use the notation from Theorem 2.7. Setting $\mathcal{B}_{i}=\mathcal{E}$, we obtain deformations of the elliptic curves defined by the rulings $f_{i}: S_{i} \rightarrow B_{i}$. Next, we deform the ramification locus $C \subset S$. Set $\mathcal{C}_{i}=\mathcal{E}$ for $i=1$ and $i=n$, and $\mathcal{C}_{i}=\mathcal{E} \amalg \mathcal{E}$ for $2 \leq i \leq n-1$. Let $f_{n}: \mathcal{C}_{n} \rightarrow \mathcal{B}_{n}$ be the double covering defined by $2: \mathcal{E} \rightarrow \mathcal{E}$. For $2 \leq i \leq n-1$, let $f_{i}: \mathcal{C}_{i} \rightarrow \mathcal{B}_{i}$ be the disjoint union of the identity on $\mathcal{E}$. Now the family of geometrically ruled surfaces $\overline{\mathcal{S}}_{i}=\mathbb{P}\left(\left(f_{i}\right)_{*}\left(\mathcal{O}_{\mathcal{C}_{i}}\right)\right)$ is a deformation of the minimal models $\bar{S}_{i}$. Lifting the centers for the blowing-up in $S_{i} \rightarrow \bar{S}_{i}$, you obtain a deformation $\mathcal{S}$ of $S$. Deforming the gluing map $\varphi: C \rightarrow D$ and making a gluing over $A$, we obtain a flat family $\mathfrak{X} \rightarrow \operatorname{Spec}(A)$ of type II Enriques surfaces with special fiber $\mathfrak{X}_{\sigma}=X$, such that $\mathfrak{X}_{\eta}$ is an ordinary Enriques surface of type II.

To obtain classical surfaces as generic fibers, we have to replace the projection $f_{n}: \mathcal{C}_{n} \rightarrow \mathcal{B}_{n}$ defined in the previous construction as $2: \mathcal{E} \rightarrow \mathcal{E}$ with the dual projection $2^{*}: \operatorname{Pic}_{\mathcal{E} / A}^{0} \rightarrow \operatorname{Pic}_{\mathcal{E} / A}^{0}$, which has kernel $G_{0, t}$. 
Proof of Theorem 6.1. Set $A_{1}=k\left[t_{1}\right] /\left(t_{1}^{2}\right)$, and let $X_{1}$ be a first-order deformation of $X$ towards ordinary Enriques surfaces obtained from Lemma 6.4 by restriction. Then the relative Picard scheme is $\operatorname{Pic}_{X_{1} / A_{1}}^{\tau}=G_{0, t_{1}}$. Similarly, set $A_{2}=k\left[t_{2}\right] /\left(t_{2}^{2}\right)$, and let $X_{2}$ be the first-order deformation of $X$ towards classical Enriques surfaces. Then $\mathrm{Pic}_{X_{2} / A_{2}}^{\tau}=G_{t_{2}, 0}$.

Seeking a contradiction, we assume that the log deformation functor $\mathrm{LD}_{X \dagger}$ is formally smooth. Setting $A=k\left[t_{1}, t_{2}\right] /\left(t_{1}^{2}, t_{2}^{2}\right)$, we can find a deformation $\mathfrak{X}$ over $A$ extending both $X_{1}$ and $X_{2}$. Consider the relative Picard scheme $G=\operatorname{Pic}_{\mathfrak{X} / A}^{\tau}$. Its closed fiber is $G \otimes k=G_{0,0}$. By Nakayama's Lemma, $G$ is a closed subgroup scheme of $G_{a, b}$ for some $a, b \in A$ with $a b=0$.

By construction, $G_{a, b} \otimes A_{1}=G_{0, t_{1}}$. Calculating modulo $t_{2}$, we obtain $a \equiv 0$ and $b \equiv \lambda_{1} t_{1}$ for some unit $\lambda_{1} \in k$. Similarly, $G_{a, b} \otimes A_{2}=G_{t_{2}, 0}$. Calculating modulo $t_{1}$, we have $b \equiv 0$, and $a \equiv \lambda_{2} t_{2}$ for some unit $\lambda_{2} \in k$. This gives $a=\lambda_{2} t_{2}$ and $b=\lambda_{1} t_{1}$, contradicting $a b=0$.

\section{Smoothings AND LIFTINGS}

Let $X$ be a proper scheme over a field $k$ of characteristic $p=2$. We shall say that $X$ is smoothable if there is an integral local noetherian $\operatorname{ring} A$ with residue field $k$, together with a proper flat $A$-scheme $Y$ with closed fiber $Y_{\sigma}=X$ and smooth generic fiber. Furthermore, we say that $X$ lifts to characteristic zero if there is an integral local noetherian ring $A$ of mixed characteristic with residue field $k$, together with a proper flat $A$-scheme $Y$ with closed fiber $Y_{\sigma}=X$. Now we come to the main result of this paper.

Theorem 7.1. Nonsmooth d-semistable Enriques surfaces with normal crossings are smoothable and lift to characteristic zero.

Proof. Fix such an Enriques surface $X$ and choose a log structure $X^{\dagger}$ of semistable type. Let $W$ be a discrete valuation ring of mixed characteristic with residue field $k$. According to [15, Theorem 8.7, the corresponding log deformation functor $\mathrm{LD}_{X^{\dagger}}:\left(\operatorname{Art}_{W[[T]]}\right) \rightarrow($ Set $)$ admits a hull $h: \mathfrak{X}^{\dagger} \rightarrow \operatorname{Spf}\left(R^{\dagger}\right)$. Here $R$ is a complete local noetherian $W[[T]]$-algebra, and $\mathfrak{X}^{\dagger}$ is a proper formal log smooth $R^{\dagger}$-scheme. We shall proceed in five steps.

Step (i): $X$ is classical. According to Proposition 1.1, there is an ample $\mathcal{O}_{X^{-}}$ module $\mathcal{L}$. Let $X_{m} \subset \mathfrak{X}$ be the $m$-th order infinitesimal neighborhood of the closed fiber $X \subset \mathfrak{X}$. The exact sequence

$$
\operatorname{Pic}\left(X_{m+1}\right) \longrightarrow \operatorname{Pic}\left(X_{m}\right) \longrightarrow H^{2}\left(X, \mathcal{O}_{X}\right)
$$

together with $H^{2}\left(X, \mathcal{O}_{X}\right)=0$ ensures that $\mathcal{L}$ extends to an invertible $\mathcal{O}_{\mathfrak{X}}$-module. Hence Grothendieck's Existence Theorem (112], Thm. 5.4.5) applies, and we conclude that $\mathfrak{X}$ is algebraizable. In other words, there is a projective flat $R$-scheme $Y$ whose completion is $Y_{/ X}=\mathfrak{X}$. By Theorem 5.2, the functor $\mathrm{LD}_{X^{\dagger}}$ is formally smooth, so the $W[[T]]$-algebra $R$ is formally smooth. Hence $X$ is smoothable and lifts to characteristic zero.

Step (ii): $X$ is of type III and in minus-one-form. For an invertible $\mathcal{O}_{X}$-module $\mathcal{L}$, let $I_{\mathcal{L}} \subset R$ be the smallest ideal so that $\mathcal{L}$ admits an extension over $\mathfrak{X} \otimes R / I_{\mathcal{L}}$. Since the obstruction group $H^{2}\left(X, \mathcal{O}_{X}\right)$ is 1-dimensional, we can find an element $r_{\mathcal{L}} \in R$ contained in the maximal ideal $\mathfrak{m}_{R} \subset R$ with $I_{\mathcal{L}}=r_{\mathcal{L}} R$ (compare [8], Prop. $1.5)$. 
Claim. There is an ample $\mathcal{O}_{X}$-module $\mathcal{L}$ with $r_{\mathcal{L}} \notin \mathfrak{m}_{R}^{2}+(2, T)$.

Assume this for a moment. By Theorem 5.2, the local ring $R$ is regular. Choose $\mathcal{L}$ as in the Claim. Then the sequence $\left(2, T, r_{\mathcal{L}}\right)$ is part of a regular system of parameters. Hence the map $W[[T]] \rightarrow R / r_{\mathcal{L}} R$ admits a section. Using Grothendieck's Existence Theorem, we obtain a $\log$ smooth deformation $Y \rightarrow \operatorname{Spec}(W[[T]])$. Consequently, $X$ is smoothable and lifts to characteristic zero. Note that the restriction of $Y$ over $k[[T]]$ has smooth total space, and that the restriction over $W$ is a locally trivial deformation.

It remains to prove the Claim. Since $X$ is in minus-one-form, Proposition 3.1 ensures that $X$ is projective. Seeking a contradiction, we assume that $r_{\mathcal{L}} \in \mathfrak{m}_{R}^{2}+$ $(2, T)$ for all ample $\mathcal{O}_{X}$-modules $\mathcal{L}$. This means that $\operatorname{Pic}(Y) \rightarrow \operatorname{Pic}(X)$ is surjective for all locally trivial first-order $\log$ smooth deformations $Y^{\dagger} \rightarrow \operatorname{Spec}\left(k[\epsilon]^{\dagger}\right)$. By Serre duality and $\omega_{X}=\mathcal{O}_{X}$, the Yoneda pairing

$$
H^{1}\left(X, \Omega_{X^{\dagger} / k^{\dagger}}^{1}\right) \times H^{1}\left(X, \mathcal{H o m}\left(\Omega_{X^{\dagger} / k^{\dagger}}^{1}, \mathcal{O}_{X}\right)\right) \longrightarrow H^{2}\left(X, \mathcal{O}_{X}\right)
$$

in nondegenerate. A first-order locally trivial $\log$ deformation $Y^{\dagger}$ corresponds to an element $\zeta^{\dagger} \in H^{1}\left(\mathcal{H o m}\left(\Omega_{X^{\dagger} / k^{\dagger}}^{1}, \mathcal{O}_{X}\right)\right)$. Its image $\zeta \in H^{1}\left(\mathcal{H o m}\left(\Omega_{X / k}^{1}, \mathcal{O}_{X}\right)\right)$ corresponds to the underlying deformation $Y$. According to [21, Lemma 6.5, the obstruction map $\partial$ in

$$
\operatorname{Pic}(Y) \longrightarrow \operatorname{Pic}(X) \stackrel{\partial}{\longrightarrow} H^{2}\left(X, \mathcal{O}_{X}\right)
$$

factors over

$$
\operatorname{Pic}(X) \stackrel{\text { dlog }}{\longrightarrow} H^{1}\left(X, \Omega_{X}^{1}\right) \stackrel{\langle\cdot, \zeta\rangle}{\longrightarrow} H^{2}\left(X, \mathcal{O}_{X}\right) .
$$

Since $\operatorname{Pic}(X)$ extends over all such deformations $Y$, we infer that the composition

$$
\operatorname{Pic}(X) \stackrel{\text { dlog }}{\longrightarrow} H^{1}\left(X, \Omega_{X}^{1}\right) \longrightarrow H^{1}\left(X,\left(\Omega_{X / k}^{1}\right)^{\vee \vee}\right) \longrightarrow H^{1}\left(X, \Omega_{X^{\dagger} / k^{\dagger}}^{1}\right)
$$

must be zero. The commutative diagram in (3) gives a commutative diagram

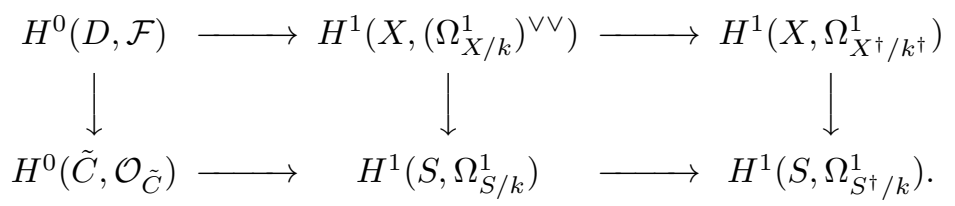

with $\mathcal{F}=\operatorname{kern}\left(\mathcal{O}_{\tilde{D}} \rightarrow \mathcal{O}_{T}\right)=\operatorname{cokern}\left(\left(\Omega_{X / k}^{1}\right)^{\vee \vee} \rightarrow \Omega_{X^{\dagger} / k^{\dagger}}^{1}\right)$. Since the map $\operatorname{Pic}(X) \rightarrow H^{1}\left(X, \Omega_{X^{\dagger} / k^{\dagger}}^{1}\right)$ is zero, the image of $\operatorname{Pic}(X) \rightarrow H^{1}\left(S, \Omega_{S / k}^{1}\right)$ is contained in the image of $H^{0}(D, \mathcal{F}) \rightarrow H^{1}\left(S, \Omega_{S / k}^{1}\right)$.

We shall derive a contradiction as follows. Since $X$ is in minus-one-form, the invertible $\mathcal{O}_{S}$-module $\mathcal{O}_{S}(C)$ descends to an invertible $\mathcal{O}_{X}$-module $\mathcal{L}$ as in the proof of Proposition 3.1 Hence the Hodge class of $C$ is of the form $\sum \lambda_{j} C_{j}$ for certain coefficients $\lambda_{j} \in k$ coming from $H^{0}(D, \mathcal{F})$. The latter condition means $\lambda_{j}=\lambda_{k}$ if the intersection $\varphi\left(C_{j}\right) \cap \varphi\left(C_{k}\right)$ is a double curve, and $\lambda_{j}+\lambda_{k}=\lambda_{l}$ if the intersection $\varphi\left(C_{j}\right) \cap \varphi\left(C_{k}\right) \cap \varphi\left(C_{l}\right)$ is a triple point. According to Proposition 3.3, there are at least two components, say $S_{0}, S_{1} \subset S$, with $\lambda_{j}=1$ for all $C_{j} \subset S_{0} \cup S_{1}$. Using the double curve and triple point conditions, we infer that the multiplicities in $\sum \lambda_{j} C_{j}$ near $S_{0}$ are as follows: 


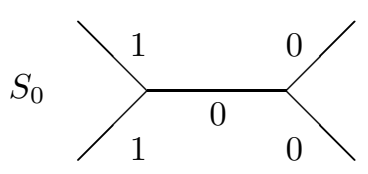

Inductively, we infer that the multiplicities $\lambda_{j}$ on each component $S_{i} \neq S_{0}$ are cycles of the form $1,0,0,1,0,0, \ldots 1,0,0$. On the other hand, $\lambda_{j}=1$ for all $C_{j} \subset S_{1}$, contradiction.

Step (iii): $X$ is of type III. By Proposition 3.2, there is a sequence of type I and type II modifications putting $X$ into minus-one-form $X^{\prime}$. According to case (ii), there is a smoothing of $X^{\prime}$ with smooth total space and a locally trivial deformation to characteristic zero. Applying the reverse sequence of modifications to the total space of these deformations, we easily obtain the desired deformations of $X$.

Step (iv): $X$ is ordinary of type II. The arguments are similar to the case of type III surfaces in minus one form, so I leave them as an exercise.

Step $(v): X$ is supersingular. Set $A=k[[t]]$. According to Lemma 6.4, there is a flat projective morphism $Y \rightarrow \operatorname{Spec}(A)$ with closed fiber $Y_{\sigma}=X$, so that the generic fiber $Y_{\eta}$ is a d-semistable classical Enriques surface. Choose a closed

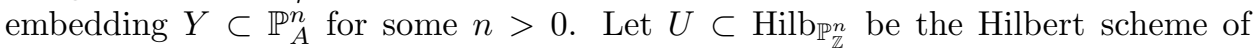
all normal crossing Enriques surfaces contained in $\mathbb{P}_{\mathbb{Z}}^{n}$. We have just constructed a curve $\operatorname{Spec}(A) \subset U$. By case (i), the point $u \in U$ representing the generic fiber $Y_{\eta}$ admits generizations corresponding to smooth Enriques surfaces and surfaces in characteristic zero. Consequently, $X$ is smoothable and lifts to characteristic zero.

\section{REFERENCES}

[1] W. Barth, C. Peters, A. Van de Ven: Compact complex surfaces. Ergeb. Math. Grenzgebiete (3) 4, Springer, Berlin, 1984.

[2] E. Bombieri, D. Mumford: Enriques' classification of surfaces in char. p II. In: W. Baily, T. Shioda (eds.), Complex analysis and algebraic geometry, pp. 23-42. Cambridge University Press, London, 1977.

[3] E. Bombieri, D. Mumford: Enriques' classification of surfaces in char p III. Invent. Math. 35, 197-232 (1976).

[4] M. Borelli: Divisorial varieties. Pac. J. Math. 13, 378-388 (1963).

[5] N. Bourbaki: Algèbre commutative. Chapitres 8 et 9. Masson, Paris, 1983.

[6] F. Cossec, I. Dolgachev: Enriques surfaces I. Prog. Math. 76. Birkhäuser, Boston, 1989.

[7] P. Deligne, M. Rapoport: Les schémas de modules de courbes elliptiques. In: P. Deligne, W. Kuyk (eds.), Modular Functions of one Variable II, pp. 143-316. Lect. Notes Math. 349. Springer, Berlin, 1973.

[8] P. Deligne: Relèvement des surfaces K3 en caractéristique nulle. In: J. Giraud, L. Illusie, M. Raynaud (eds.), Surface algébrique, pp. 58-79. Lect. Notes Math. 868. Springer, Berlin, 1981.

[9] R. Friedman: Global smoothings of varieties with normal crossings. Ann. Math. (2) 118, 75-114 (1983).

[10] T. Fujita: Semipositive line bundles. J. Fac. Sci. Univ. Tokyo 30, 353-378 (1983).

[11] A. Grothendieck: Éléments de géométrie algébrique II: Étuede globale élémentaire de quelques classes de morphismes. Publ. Math., Inst. Hautes Étud. Sci. 8 (1961).

[12] A. Grothendieck: Éléments de géométrie algébrique III: Étuede cohomologique des faiscaux cohérent. Publ. Math., Inst. Hautes Étud. Sci. 11 (1961).

[13] A. Grothendieck: Esquisse d'un programme. In: L. Schneps (ed.) et al., Geometric Galois actions. 1. Around Grothendieck's "Esquisse d'un programme", pp. 5-48. Lond. Math. Soc. Lect. Note Ser. 242. Cambridge University Press, Cambridge, 1997. 
[14] L. Illusie: Logarithmic spaces (according to K. Kato). In: V. Cristante (ed.) et al., Barsotti symposium in algebraic geometry, pp. 183-203 Perspect. Math. 15. Academic Press, San Diego, 1994.

[15] F. Kato: Log smooth deformation theory. Tohoku Math. J. (2) 48, 317-354 (1996).

[16] K. Kato: Logarithmic structures of Fontaine-Illusie. In: J.-I. Igusa (ed.) et. al., Algebraic analysis, geometry, and number theory, pp. 191-224. Johns Hopkins Univ. Pr., Baltimore, 1989.

[17] Y. Kawamata, Y. Namikawa: Logarithmic deformations of normal crossing varieties and smoothing of degenerate Calabi-Yau varieties. Invent. Math. 118, 395-409 (1994).

[18] V. Kulikov: Degenerations of K3 surfaces and Enriques surfaces. Math. USSR, Izv. 11, 957-989 (1977).

[19] R. Miranda, D. Morrison: The minus one theorem. In: R. Friedman, D Morrison (eds.), The birational geometry of degenerations, pp. 173-259. Prog. Math. 29. Birkhäuser, Basel, 1983.

[20] D. Mumford: Enriques' classification of surfaces in char p I. In: D. Spencer, S. Iyanaga (eds.), Global Analysis, pp. 325-339. Princeton University Press, Princeton, 1969.

[21] I. Nakamura: On moduli of stable quasi abelian varieties. Nagoya Math. J. 58, 149-214 (1975).

[22] U. Persson: On degenerations of algebraic surfaces. Mem. Am. Math. Soc. 189 (1977).

[23] M. Raynaud: Specialisation du foncteur de Picard Publ. Math., Inst. Hautes Étud. Sci. 38, 27-76 (1970).

[24] J. Silverman: The arithmetic of elliptic curves. Grad. Texts Math. 106. Springer, Berlin, 1985.

[25] J. Tate: Finite flat group schemes. In: G. Cornell, J. Silverman, G. Stevens (eds.), Modular forms and Fermat's last theorem. Springer, New York, 1995.

Mathematische Fakultät, Ruhr-Universität, 44780 Bochum, Germany

Current address: M.I.T. Department of Mathematics, 77 Massachusetts Avenue, Cambridge MA 02139-4307, USA

E-mail address: s.schroeer@ruhr-uni-bochum.de 